\title{
SPECTROSCOPY WITH TRAPPED FRANCIUM
}

\author{
LUIS A. OROZCO \\ Department of Physics and Astronomy \\ State University of New York \\ Stony Brook NY 11794-3800 \\ United States
}

\begin{abstract}
These notes present a review of the francium experiments at Stony Brook. The measurements show that the level of quantitative understanding of the atomic structure is sufficient to pursue experiments in atomic parity non-conservation.
\end{abstract}

\section{Introduction}

Since the pioneering work of the Bouchiats (see review [1] and the book of Khriplovich [2]) on atomic parity non conservation(PNC) in heavy elements, efforts have centered in atoms with atomic number greater than fifty $[3,4,5,6,7]$. Recent measurements in Cs [3] have reached sensitivity to the nuclear spin dependent part of the interaction (primarily due to the nuclear anapole moment), opening a new avenue for studies of the weak interaction in the nucleus $[8,9]$, an area otherwise very difficult to probe.

On the other hand, the spin independent measurements, helped by the $a b$ initio atomic physics calculations $[11,10]$ can be used to put constraints on physics beyond the Standard Model [12]. Recent analysis of the results $[13,14,15]$ show that an atomic PNC measurement can place limits in the masses of extra neutral gauge bosons. However, the atomic and nuclear theory calculations are sufficiently complicated that very high order corrections previously neglected [16] can modify the high energy limits obtained from the atomic PNC results.

The anapole moment of a nucleus is a parity violating, time reversal conserving moment that arises from parity non-conserving weak interactions between the nucleons $[1,2,17]$. It can only be detected in a PNC electron-nucleus interaction. 
For the extraction of weak force information from an atomic PNC experiment it is necessary to have excellent knowledge of the atomic structure. In this notes we show that at Stony Brook our measurements [18, 19, 20, 21] and the present atomic structure calculations of $\operatorname{Fr}[22,23,24,25]$ are consistent within their claimed accuracy of $1 \%$. This is paving the way towards a PNC measurement in Fr that will give information about the weak force, in particular about the anapole moment.

\section{Francium production and trapping}

Francium is the heaviest of the alkali elements. It is the most unstable of the first one hundred and three elements since it longest lived isotope has a lifetime of only twenty two minutes. It is now possible to perform precise measurements of the atomic properties of francium thanks to two different developments. First new targets are available for its production through nuclear fusion reactions, and second the advent of laser trapping and cooling techniques for on-line capture of the radioactive isotopes produced. Fr has many properties that make it an excellent candidate for measurements of parity violation effects in atoms. The interested reader should look for the contributions that treat laser cooling and trapping topic in this book as well as in other textbooks. We have a review article that focuses on radioactive atoms [26]. The doctoral dissertations of Gerald Gwinner [27] and John E. Simsarian [28] should also be of great help for those interested in this topic.

\subsection{HISTORY}

Marguerite Perey [29], of the Curie Institute in Paris, reported in 1939 the discovery of a new radioactive element. While doing chemical analysis of the decay products of actinium, she found an element that behaved as an alkali. She named the new element francium after her country.

In the last two decades, starting with the observation of the $\mathrm{D}_{2}$ line of francium [30], the energies of states accessible by laser excitation were measured in pioneering work at ISOLDE [31]. Figure 1 shows a rough time-line of the experimental work in francium. Studies at ISOLDE of the hyperfine splittings revealed information about the nuclear structure of the francium isotopes from 207 to 213 and 220 to 228 [32]. Andreev et al. [33] studied several Rydberg levels and the ionization potential using ${ }^{221} \mathrm{Fr}$, a daughter product of the decay of ${ }^{229} \mathrm{Th}$, by laser-resonance ionization.

We successfully trapped francium in a magneto optical trap (MOT) in September of 1995 [34]. That was the beginning of a systematic study of its atomic properties including lifetimes, hyperfine splittings and energy levels. The purpose has been to characterize the atom as best as we can and compare our measurements with atomic and nuclear structure calculations. 


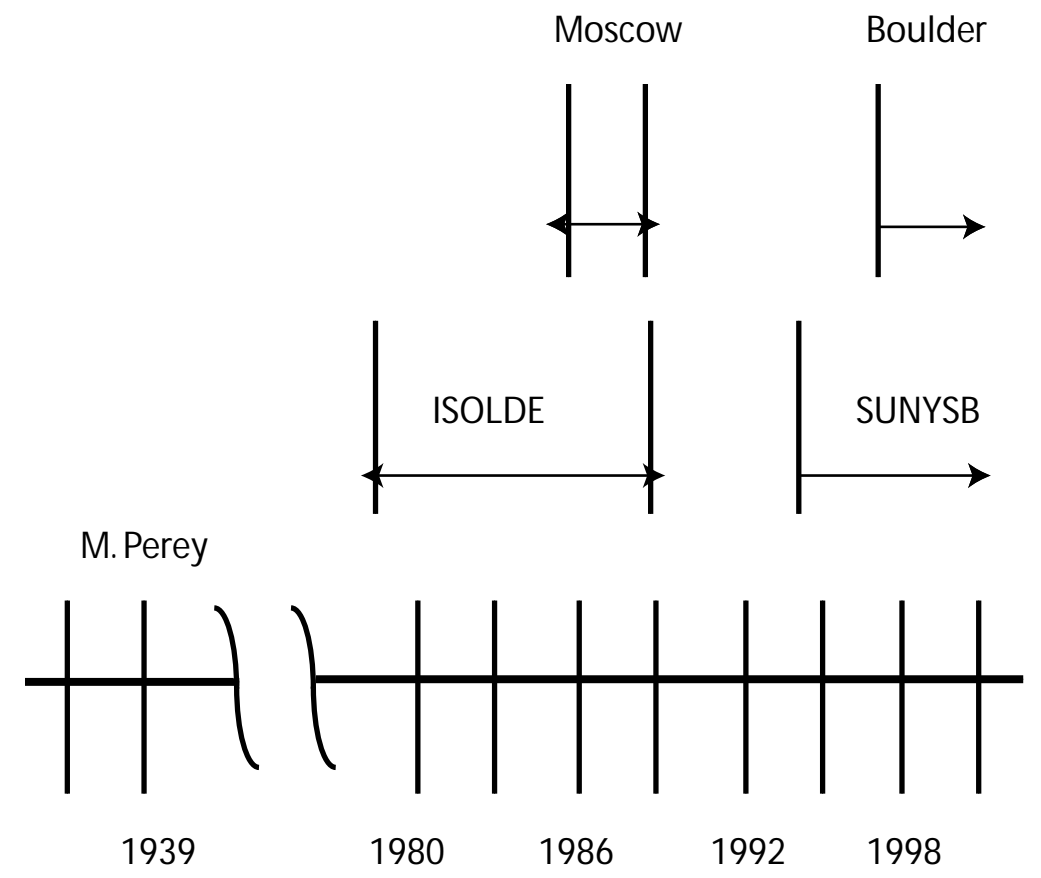

Figure 1. Time line of Fr work.

A different approach to francium trapping has also been successful [35]. The collaboration of Lawrence Berkeley Laboratory and The University of Colorado uses a radioactive source that produces the neutron rich ${ }^{221} \mathrm{Fr}$ isotope. It is an important achievement and it complements the work at Stony Brook because of the ability to trap neutron rich isotopes. The Colorado group has developed very sensitive spectroscopic methods for the detection of Fr.

\subsection{FRANCIUM PRODUCTION}

We have found a way to produce enough amounts of francium on line in an accelerator using as a guide the work at ISOLDE. We have to make the atoms using a nuclear fusion reaction in a target. The projectiles have energies around $100 \mathrm{MeV}$. The products come to a stop in the target. As the target is heated, the reaction products diffuse to the surface, are surfaceionized and are transported as ions to a region where after neutralization they are trapped in a MOT for further measurements. Figure 2 shows the energy scales used for experiments in francium.

The apparatus used to trap francium is based on the same principles as the one used to trap radioactive ${ }^{79} \mathrm{Rb}$ at Stony Brook [36]. Beams of ${ }^{18} \mathrm{O}$ 


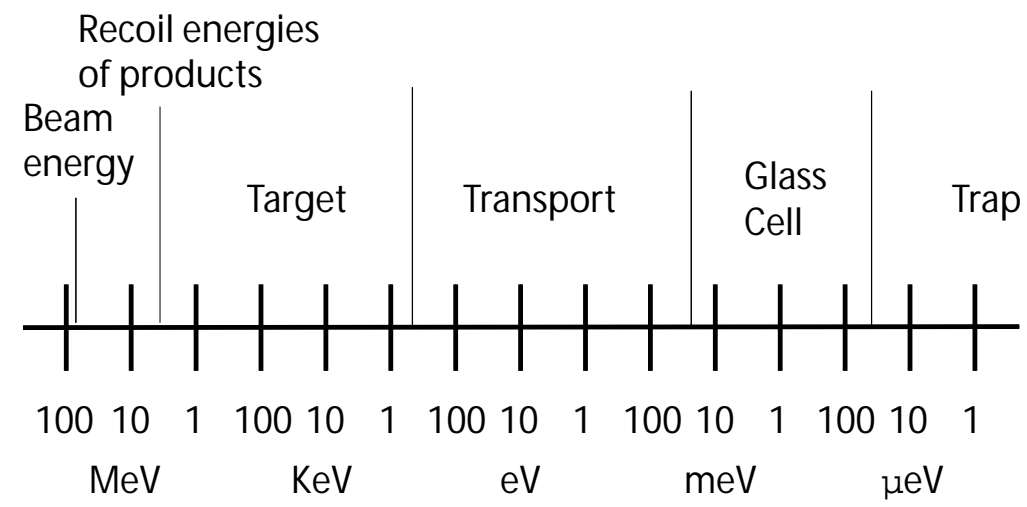

Figure 2. Energy thermometer for production, trapping and spectroscopy of Fr .

from the Stony Brook superconducting LINAC are incident on a Au target mounted on a $\mathrm{W}$ rod. The ${ }^{197} \mathrm{Au}\left({ }^{18} \mathrm{O}, \mathrm{x} n\right)$ reaction at $100 \mathrm{MeV}$ produces predominantly ${ }^{210} \mathrm{Fr}$, which has a $3.2 \mathrm{~min}$ half-life. ${ }^{210} \mathrm{Fr}$ has an estimated $\alpha$ decay branching of $60 \pm 30 \%$ with the remaining decays by $\beta^{+}$or electron capture. The target is heated to $\approx 1200 \mathrm{~K}$ by the beam power and by an auxiliary resistance heater as seen in Fig. 3. The francium surface ionizes as it escapes from the gold because the work function of gold $(4.8-5.1 \mathrm{eV})$ is larger than the ionization potential of francium $(4.07 \mathrm{eV})$.

A beam energy of $100 \mathrm{MeV}$ is optimal for production of ${ }^{210} \mathrm{Fr}$ since this isotope is created very close to the surface facilitating its escape from the target. Changing the beam energy selects the production rate of the francium isotopes. Increasing the beam energy allows the francium nucleus to expel more neutrons during the cooling process following nuclear fusion. This produces francium isotopes with fewer neutrons closer to the gold surface. We have observed traps of ${ }^{208,209,210,211,212} \mathrm{Fr}$. To produce and trap ${ }^{212} \mathrm{Fr}$, we use an isotopically enriched $\mathrm{Pt}$ target [37]. Its $19.6 \mathrm{~m}$ half life makes it nearly the longest lived francium isotope.

The francium ions enter the ion transport system shown in Fig. 3. The target and electrodes are cylindrically symmetric to reduce aberrations, and the ions are extracted at $135^{\circ}$ from the ${ }^{18} \mathrm{O}$ beam direction. We measure the number of Fr ions in the beam by stopping them on a retractable plate viewed by a silicon surface-barrier detector. We determine the number of francium atoms from the alpha-particle activity and the known solid angle of the detector. The different alpha energies and lifetimes allow isotope identification. A beam from the Stony Brook Superconducting LINAC of $6.3 \times 10^{11}{ }^{18} \mathrm{O}$ ions $/ \mathrm{s}$ on the $\mathrm{Au}$ produces $\approx 1 \times 10^{6} / \mathrm{s}{ }^{210} \mathrm{Fr}$ ions $/ \mathrm{s}$ at the catcher.

Close to the melting point of gold $(1336 \mathrm{~K})$ the Fr diffuses the most 


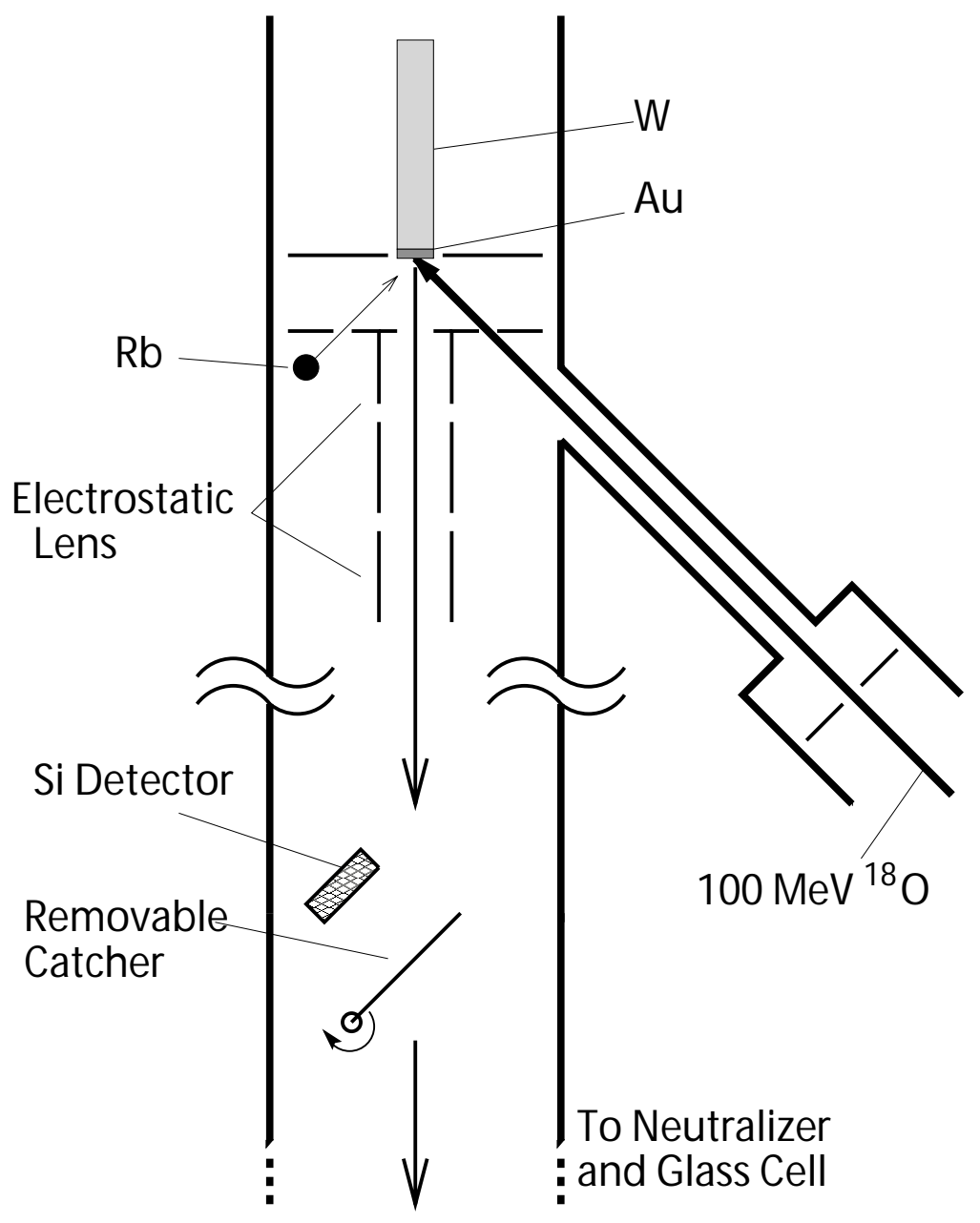

Figure 3. Schematic view of target and ion transport system.

rapidly but the structural properties of the Au may become unstable. The coil and beam current heat the target and we have found a sensitive dependence of the number of francium ions on the target temperature. The elevated temperature is necessary for the alkali elements to rapidly diffuse to the surface and be surface ionized [38]. We have repeatedly observed a sharp increase in the number of francium ions that escape from a new gold target when it is heated. Fig. 4 plots the alpha decay rate of francium ions at the silicon detector as a function of time while the beam is on the target. We have observed that increasing the ${ }^{18} \mathrm{O}$ beam current prior to the sharp transition has little effect on the francium escape rate (see Fig. 4b). Once the transition is reached, a large increase in the francium escape rate oc- 


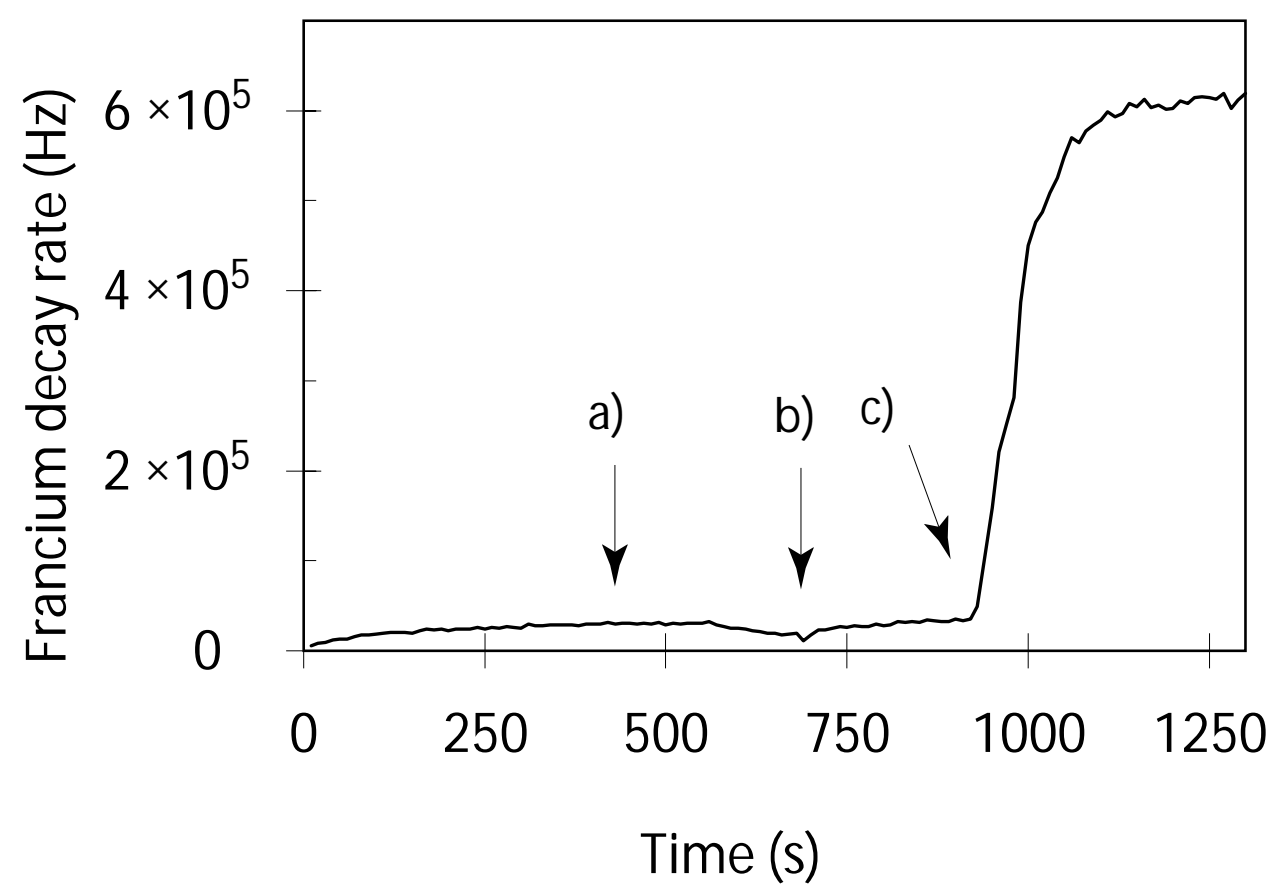

Figure 4. Francium alpha-particle decay rate at the catcher in the ion transport system versus time. The decay rate reaches an equilibrium at point a). Increasing the beam current has little effect on the production rate at point b). At point $c$ ), the target heating is increased and the transition occurs.

curs (see Fig. 4c). While we do not completely understand this behavior, it seems that the target may be locally melting at the point where the beam hits the gold. However, the beam does not fully penetrate the gold foil as there is no hole in the surface. The transition can be repeated with a single target although the increase is less dramatic after several experiments. The behavior may be caused by the removal of impurities from the gold surface that prevent surface ionization of the francium. Images taken of the new target show the region of melting by changes in the emissivity of the surface. Such changes always coincide with the increase in alpha production.

The apparatus shown in Fig. 5 separates the production and the trapping regions. This is critical to operate the trap in a ultra-high vacuum environment. Extracted at around $800 \mathrm{~V}$, the francium ions travel through three sets of electrostatic lenses that focus the ions and three sets of electrostatic plates steer them. The ion transport system is all electrostatic to be mass independent. This allows us to fine tune it with a Rb beam generated by the dispenser next to the target shown in Fig. 3. After about one meter, the ions pass through a differential-pumping aperture to the region 


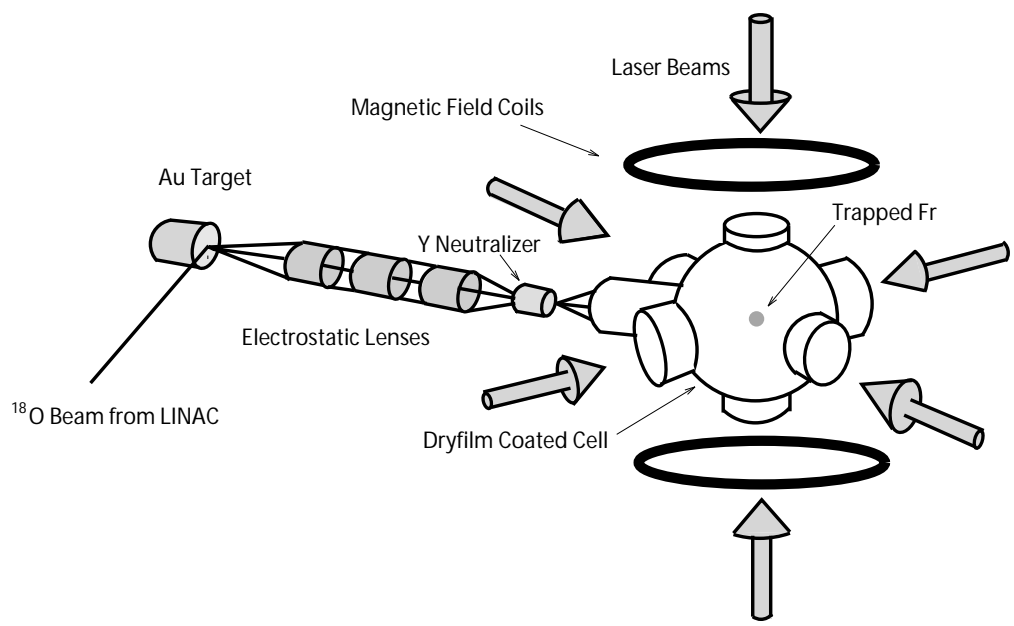

Figure 5. Schematic view of target, ion transport system, and magneto optical trap.

of ultra-high MOT vacuum. The ions are deposited on the inner surface of a cylinder coated with yttrium and heated to $970 \mathrm{~K}$ (melting point of $\mathrm{Y}$ $=1752 \mathrm{~K})$. The low work function of $\mathrm{Y}(3.1 \mathrm{eV})$ results in the release of neutral Fr atoms from the $\mathrm{Y}$ surface that form an atomic beam directed towards the vapor cell MOT.

At present we can not be in the target room, where the trapping happens, since there is a high level of neutrons released in the heavy-ion fusion reaction that produces francium. We have finished the construction of a new francium production and transport system that permits working with the trapped atoms in a radiation free environment. We have tested the new target and begun to understand its performance. The trap incorporates many of the ideas that have been generated by the Triumf and Los Alamos groups.

\subsection{THE FRANCIUM TRAP}

We use a vapor cell MOT [39]. This requires special attention to the glass utilized for the vapor cell. It does not require any deceleration of the Fr atoms before they enter the glass cell, such as that provided by a Zeeman decelerator [40].

\subsubsection{Implementation of the magneto-optic trap}

The francium trap consists of a $10 \mathrm{~cm}$ diameter Pyrex bulb with six $5 \mathrm{~cm}$ diameter windows and two viewing windows $3 \mathrm{~cm}$ in diameter. The MOT is formed by six intersecting laser beams each with $1 / e^{2}$ (power) diameter 
of $4 \mathrm{~cm}$ and typical intensity of $7.8 \mathrm{~mW} / \mathrm{cm}^{2}$, and a magnetic field gradient of $5.5 \times 10^{-2} \mathrm{~T} / \mathrm{m}$. The magnetic field gradient has been calculated from the equations in the article by Bergeman et al. [41]. The glass cell is coated with a non-stick Dry-film coating $[42,43]$ to allow the atoms multiple passes through the trapping region after thermalization with the walls [39].

\subsubsection{The Problem of the vapor cell}

Atoms leave the neutralizer in a beam and enter the vapor cell made of pyrex (shown in figure 5). The vapor-cell approach relies on the rethermalization of atoms interacting with the container walls, replenishing the low-velocity Maxwell tail from which the MOT can trap. On bare silica, pyrex, or metal surfaces, alkalis stick with a probability close to one. This is due to two effects: Chemisorption where the alkali atom undergoes a chemical reaction on the wall and is permanently removed from the vapor, and physisorption where the atom is trapped in a potential well on the surface for an average time $\bar{\tau}_{s}$ and returns afterwards into the vapor. This results in a reduced vapor pressure inside the bulb.

Once a monolayer of alkali atoms has formed on the walls, the vapor pressure rises to its usual value. This does not pose a particular problem in experiments with stable isotopes, since a monolayer is easily applied. With production rates of radioactive alkalis of less than $10^{10} \mathrm{sec}^{-1}$ it is impossible to form such a monolayer $\left(\approx 10^{16}\right.$ atoms on $100 \mathrm{~cm}^{2}$ surface). On the other hand calculations show that for a single pass through the trapping zone the Maxwell tail amounts typically only to less than 1/1000 of all atoms. The trapping efficiency is very low. For $10^{5}$ atoms entering the cell at most 20 end up trapped. The solution is to find a surface coating which reduces chemisorption and physisorption. Such coatings have been investigated for use in hydrogen masers, Cs/Rb frequency standards, and polarized targets $[44,45]$.

Teflon is a well known coating, used e.g. in hydrogen masers. However, alkalis are among the few substances that react with it. Ramsey et al. [44] report that after approximately 10 wall collisions Cs sticks permanently. An alternative are hydrocarbon surfaces, such as Dri-Film, polyethylene, and paraffin. When properly applied, these chemicals will form methyl groups on the host surface in such a way that the hydrogen atoms shield the incoming alkali atom from getting close and reacting with $\mathrm{C}, \mathrm{Si}$, or $\mathrm{O}$ atoms deeper in the surface.

These coatings display low adsorption energies and reaction rates. The results vary from group to group and are sometimes contradictory. This might be caused by the extreme sensitivity of the coatings to cleaning procedures and different container geometries. Since a MOT requires a significantly better vacuum than some of the other applications of coatings 


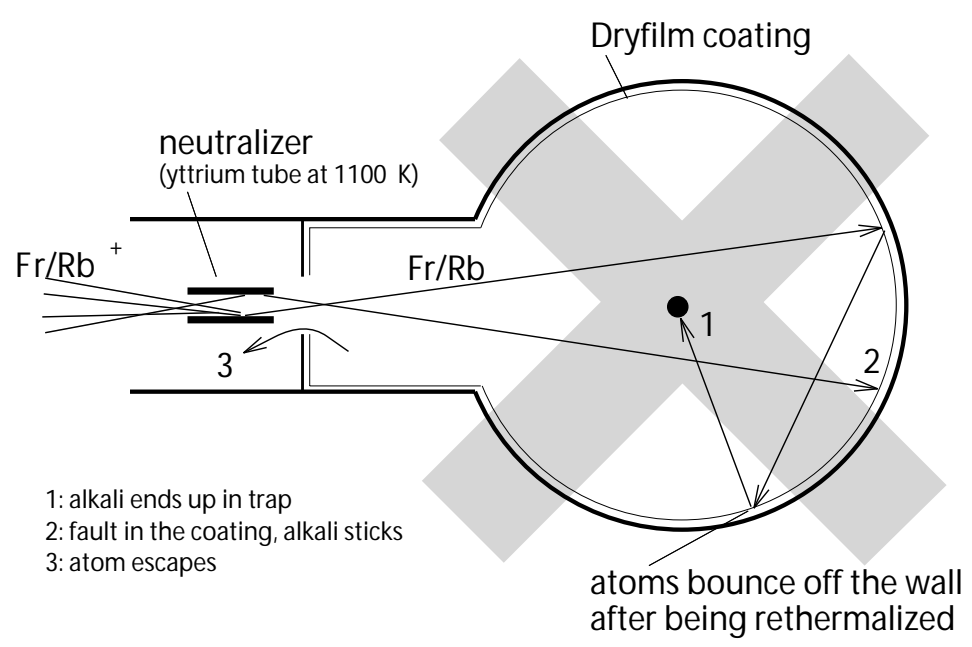

Figure 6. Simplified cross-sectional view of the vapor cell with the neutralizer.

such as masers and polarized targets, one has to pay special attention to the outgassing. Opacity and chemical reaction rates must be minimized, too. Dri-Film coating was chosen for the MOT, using the procedure of Swenson and Anderson based on SC-77 [42]. It can be baked to at least $275^{\circ} \mathrm{C}$, is usable on glass and certain metals, and was successfully tested by several groups. Figure 6 shows the different processes that can happen inside the trap.

\subsubsection{Fr trapping}

The atomic energy levels of the ${ }^{210} \mathrm{Fr}$ atom relevant for trapping are shown in Fig. 7. A titanium-sapphire (Ti:Sapph) laser excites the cycling transition $7 S_{1 / 2}, \mathrm{~F}=13 / 2 \rightarrow 7 P_{3 / 2}, \mathrm{~F}=15 / 2$ at $718 \mathrm{~nm}$. The ${ }^{210} \mathrm{Fr}$ ground-state hyperfine splitting of $46.8 \mathrm{GHz}$ requires an extra laser for repumping the atoms that fall into the $\mathrm{F}=11 / 2$ ground state. Transitions to either the $7 P_{3 / 2}$ or $7 P_{1 / 2}$ levels repump the atoms. A free-running diode laser cooled to approximately $77 \mathrm{~K}$ with liquid nitrogen repumps the atoms into the cycling transition via $7 S_{1 / 2}, \mathrm{~F}=11 / 2 \rightarrow 7 P_{3 / 2}, \mathrm{~F}=13 / 2$. A second Ti:Sapph laser or diode laser at $817 \mathrm{~nm}$ repumps the atoms on the $7 S_{1 / 2}, \mathrm{~F}=11 / 2 \rightarrow$ $7 P_{1 / 2}, \mathrm{~F}=13 / 2$ transition. We have more laser power available at $817 \mathrm{~nm}$ and typically repump the atoms with transitions to the $7 P_{1 / 2}$ level.

We have developed absolute frequency references for the trapping lasers because there are no stable isotopes of Fr to use for this purpose. The atlas of the $\mathrm{I}_{2}$ spectrum [46] provided the laser frequency calibration for the initial trapping experiment [34]. In the $718 \mathrm{~nm}$ region of the spectrum the $\mathrm{I}_{2}$ has little absorption unless we heat the cells to temperatures 


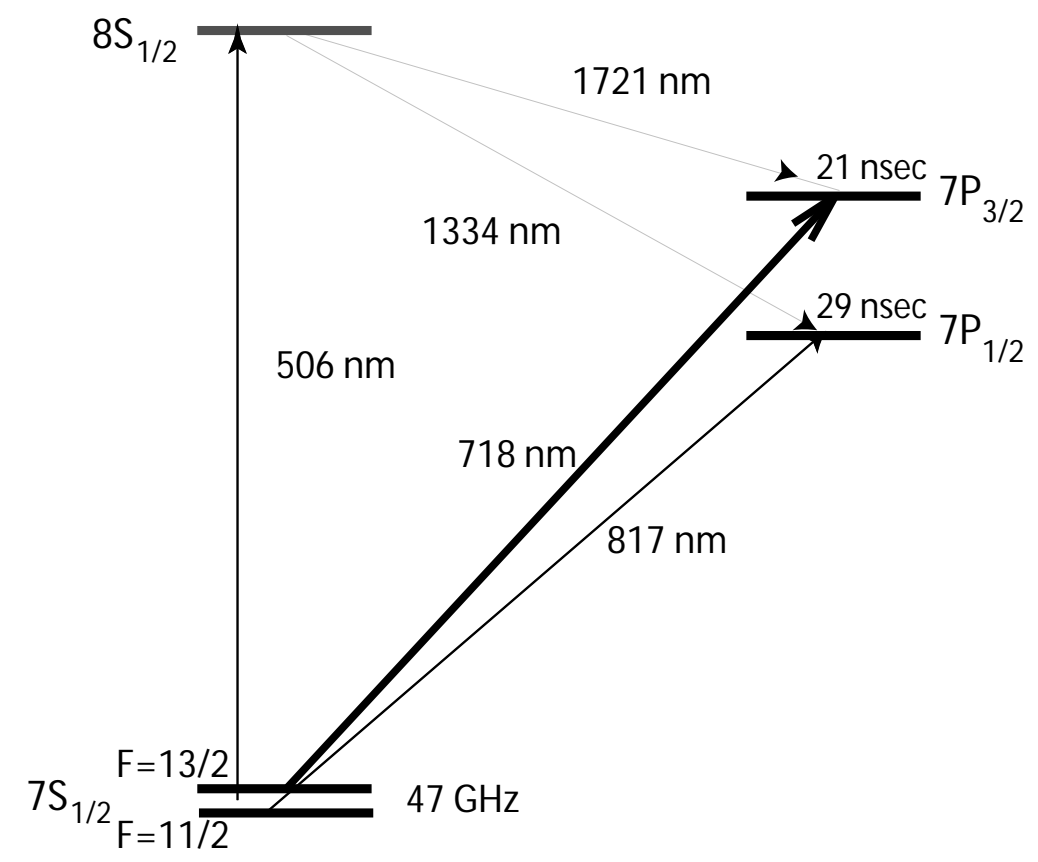

Figure \%. Relevant energy levels for trapping and the possible PNC experiment in francium.

greater than $570 \mathrm{~K}$. Then the absorption features are Doppler broadened to about $1 \mathrm{GHz}$. The tabulated line 381 is about $3.5 \mathrm{GHz}$ away from the trapping transition frequency. To make an absorption feature at the trapping frequency, we send a portion of the Ti:sapph laser beam through an electro-optic modulator to generate FM sidebands at $3.1 \mathrm{GHz}$ (see Fig. 8). The FM modulated beam passes through a series of acousto-optic modulators to shift an additional $400 \mathrm{MHz}$. It then passes through a heated $\mathrm{I}_{2}$ absorption cell so that when the lower frequency sideband is on resonance with $\mathrm{I}_{2}$ line 381 , the laser frequency is close to the trapping transition. The iodine absorption signal comes from the mixed-down photocurrent of a fast photodiode providing an absolute, but rather broad reference. Following the initial trapping of francium, we have used a wavemeter with an accuracy of $\pm 0.001 \mathrm{~cm}^{-} 1$ to locate the trapping transitions. A thermally stabilized etalon with broadband mirrors and $500 \mathrm{MHz}$ free spectral range serves as a secondary relative reference. This etalon is further stabilized against drifts by a diode laser locked to a saturation spectroscopy resonance in $\mathrm{Rb}$.

We now have a computer-controlled scanning Fabry-Perot cavity for long term control of the frequencies of all the lasers involved in the trapping and probing of francium [47]. The system works by keeping the relative 


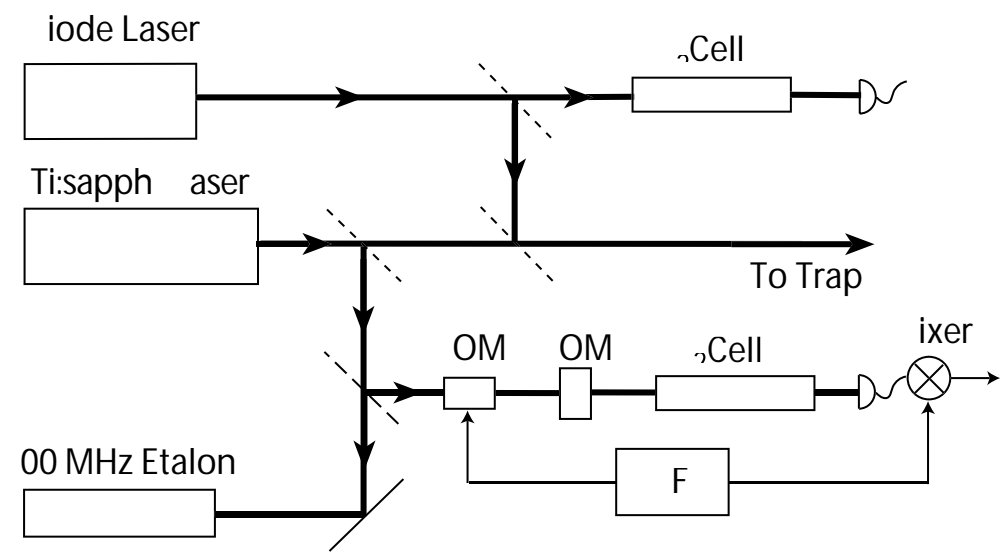

Figure 8. Block diagram of the laser system.

distance of the fringes produced by the lasers compared to the fringes from a frequency stabilized HeNe laser. We rely on the wavemeter to first find the resonances, and then we can lock and finely adjust the frequency with the cavity system.

The $718 \mathrm{~nm}$ repumper laser is modulated in frequency with an amplitude of $500 \mathrm{MHz}$. Between the $\mathrm{I}_{2}$ lines 387 and 388 of Ref. [46] there is a small non-tabulated absorption feature that matches the position of the repumping transition in ${ }^{210} \mathrm{Fr}$. We use this feature to keep the repumper laser frequency on resonance.

The hyperfine splittings of the ${ }^{210} \mathrm{Fr}$ levels of the excited state are much larger than in the other alkalis (see Fig. 7), so the expected leakage to other levels should be smaller, requiring less repumper power. The modulation depth of the trap fluorescence due to a change in the repumper frequency is uncertain in francium, so the method of repumper modulation used to detect ${ }^{79} \mathrm{Rb}$ in our previous work [36] was not feasible. Instead, the trapping laser is frequency modulated with amplitudes from 1 to $15 \mathrm{MHz}$ at a frequency of $14.5 \mathrm{kHz}$. Lock-in detection allows the rejection of the laser light scattered from the cell while measuring the fluorescence from the captured atoms. A $f / 2$ optical system collects the trap fluorescence onto a photomultiplier tube, and the signal is demodulated by a lock-in detector.

Before trapping Fr, the apparatus is tested extensively using stable ${ }^{87} \mathrm{Rb}$ injected into the cell from the dispenser next to the Au target (see Fig. 3). We use the trapped $\mathrm{Rb}$ to optimize the position of the photomultiplier that detects the trapped atoms. Then we change the laser frequencies to the francium resonances without further adjustment of the detection optics.

The energy of the $7 P_{3 / 2}$ and $7 P_{1 / 2}$ levels were measured by the ISOLDE collaboration [48]. Given the uncertainty in the location of the trapping 
transition with respect to our frequency reference, we scanned the trapping laser in the region of the resonance. We recorded the lock-in signal along with the $\mathrm{I}_{2}$ absorption spectrum and the stabilized etalon signal. With a trap lifetime of the order of 10 seconds, we chose a scan rate slow enough to allow atoms to accumulate in the trap. Figure 9 shows the fluorescence signal from the captured francium atoms. Panel (a) is a plot of the fluorescence signal of the trapped atoms as the trapping laser is scanned in frequency over a $60 \mathrm{MHz}$ range at $0.5 \mathrm{MHz} / \mathrm{s}$. The fluorescence signal was integrated with a $1 \mathrm{~s}$ time constant. The lower panels show a different, wider, scan of the laser. Panel (b) shows the fluorescence from trapped atoms. Panel (c) shows the transmission of the reference etalon. Panel (d) is the $\mathrm{I}_{2}$ absorption of the shifted sideband. For the wide scan two rates were used. Around the center of the scan we used $1.0 \mathrm{MHz} / \mathrm{s}$, with a faster one elsewhere.

The center of gravity of the trap signal is $13923.3866 \pm 0.0026 \mathrm{~cm}^{-1}$, in agreement with the calculated value of $13923.3887 \pm 0.0020 \mathrm{~cm}^{-1}$ for the ${ }^{210} \mathrm{Fr} 7 S_{1 / 2}, \mathrm{~F}=13 / 2 \rightarrow 7 P_{3 / 2}, \mathrm{~F}=15 / 2$ transition from the data by Bauche et al. [48]. The uncertainty in our measurement comes by adding in quadrature $0.0019 \mathrm{~cm}^{-1}$ from the previous calibration of line 381 [46], $0.0007 \mathrm{~cm}^{-1}$ from the fit to the shifted 381 line in our experiment, and $0.0016 \mathrm{~cm}^{-1}$ from systematics [34].

We estimate the number of atoms in the trap by comparing the measured fluorescence signal from the trap with the expected fluorescence/atom. We have signals corresponding to at least $10^{4}$ atoms. They produce good images in TV and CCD cameras. Figure 10 shows the histogram of the fluorescence from trapped Fr obtained from a CCD camera image of about $2 \times 10^{3}$ atoms. The efficiency of the process of production to capture is $10^{-3}$ coming from the ratio of trapping rate to the target production rate.

\section{Spectroscopy of $\mathrm{Fr}$}

We have studied the spectroscopy of francium in a MOT on-line with an accelerator. The captured atoms are confined for long periods of time in a small volume moving at low velocity, an ideal environment for precision spectroscopy. Our investigations have included the location of the $8 S$ and $9 S$ energy levels. The $8 S$ energy level, not observed before, is a prime candidate to use in a PNC experiment. The forbidden electric dipole transition $7 S \rightarrow 8 S$ becomes allowed through the weak force and the rate of transitions is proportional to the weak charge. We have also made measurements of radiative lifetimes in Fr. The precisions of our lifetime measurements of the $D_{1}$ and $D_{2}$ lines are comparable to those achieved in stable atoms. They tests atomic theory in a heavy atom where relativistic and correlation effects are large. 


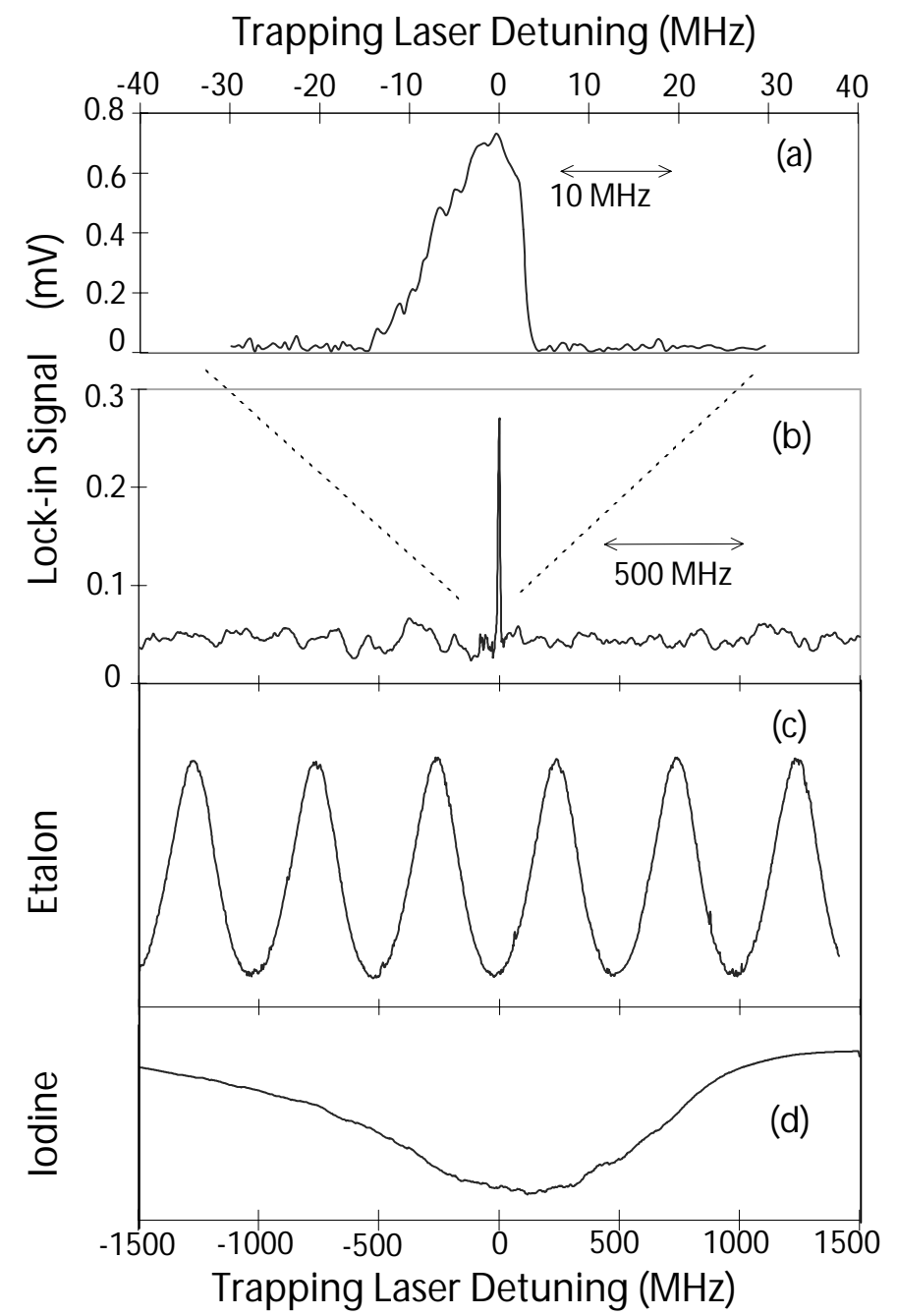

Figure 9. a) Trap fluorescence vs. frequency detuning (60 MHz scan). b) Trap fluorescence vs. frequency detuning $(3.0 \mathrm{GHz}$ scan). c) Etalon transmission with the $500 \mathrm{MHz}$ marks (3.0 GHz scan). d) $\mathrm{I}_{2}$ absorption line (3.0 GHz scan).

Atomic properties are sensitive to different ranges of the electron wave function. The energy levels are the direct eigenvalues of the wavefunctions, but do not give detailed information about the $\mathbf{r}$ dependence of the wavefunction in a particular range. The radiative lifetime, $\tau$, depends on the matrix element of the dipole moment operator, er, spatially integrated with the wave functions of the two connected levels. It is most sensitive to large $\mathbf{r}$ properties. Complementary, the hyperfine interaction probes the wave functions at the nucleus $\mathbf{r} \approx 0$. 


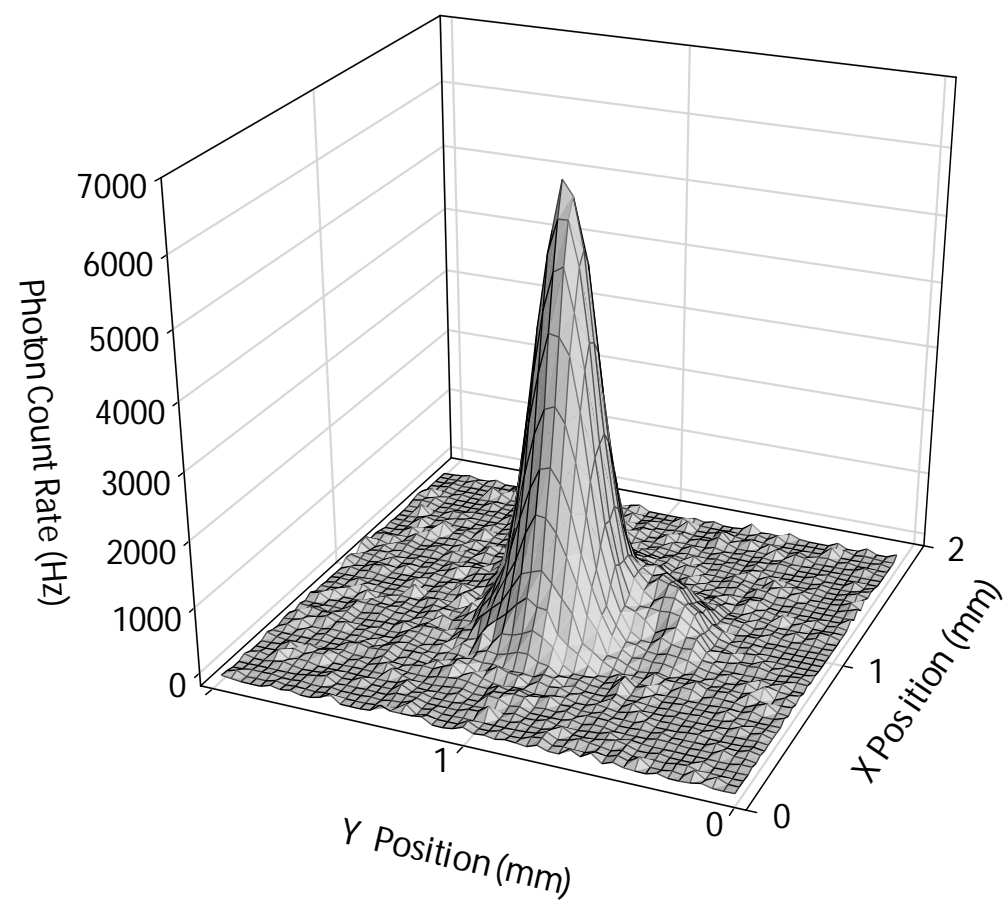

Figure 10. Two-dimensional CCD image of the fluorescence from francium atoms trapped in the MOT.

\subsection{SPECTROSCOPY OF $8 S$ AND $9 S$ LEVELS}

Although many energy levels of francium [31] have been studied, until recently the position of the two levels $(8 S$ and $9 S)$ of primary interest for PNC had not been observed. At Stony Brook we have located them. The careful engineering of the apparatus, accelerator and trapping lasers permit the experiment to run for long periods of time while maintaining a constant cloud of very cold francium atoms.

We located the 9S level [49] in 1996. Figure 11 shows the relevant levels necessary for excitation and detection. We used a two photon process to excite the atom from the $7 S_{1 / 2} \rightarrow 7 P_{3 / 2} \rightarrow 9 S_{1 / 2}$. The trapping laser provides the first photon and a narrow-linewidth diode laser operating at $851 \mathrm{~nm}$ provides the second. We looked for blue photons at $433 \mathrm{~nm}$ and 423 $\mathrm{nm}$ as the atom decayed from the $9 S$ state via the $8 P_{3 / 2}$ and $8 P_{1 / 2}$ levels. A photon counting system detected the blue photons. We also monitored the fluorescence on the cycling transition and observed that the two signals are correlated. They show the asymmetries expected from the Autler-Townes splitting of the $7 P_{3 / 2}$ level due to the intense trapping laser. Figure 12 shows the resonance detected in the count of blue photons as we scanned the 851 


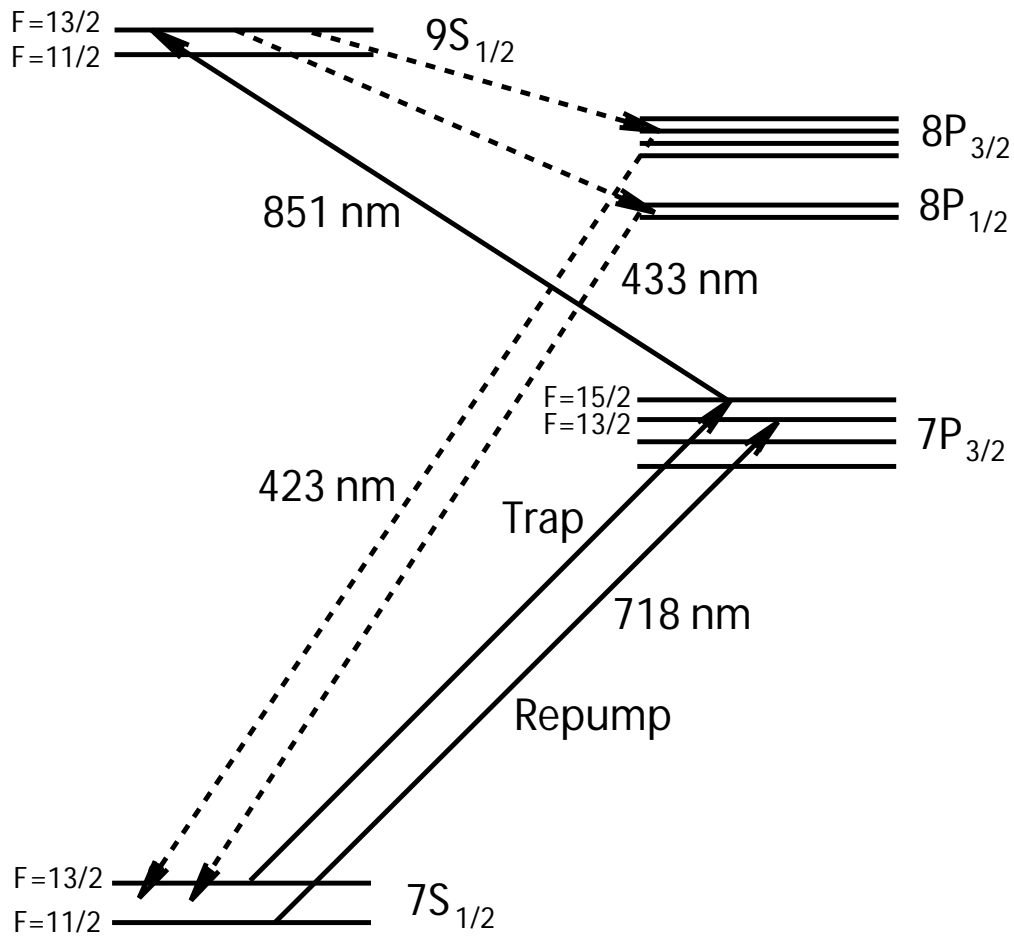

Figure 11. Diagram of energy levels of ${ }^{210} \mathrm{Fr}$ relevant for trapping and two-photon excitation of the $9 S$ level.

nm laser.

The calculations by the Dzuba et al. [22] using ab initio wave-functions for the location of the $9 S$ level were within the quoted uncertainty of $\pm 10 \mathrm{~cm}^{-1}$ accurate to four digits. In the search for this transition we were guided by the quantum defect fit to the $S$ series done by Dr. Thomas Bergeman of Stony Brook with an accuracy of $1 \mathrm{~cm}^{-1}$ in the location of the $9 S$ level. The energy difference between the $9 S_{1 / 2}$ level and the $7 S_{1 / 2}$ ground state is $25671.021 \pm 0.006 \mathrm{~cm}^{-1}$.

More important is the measurement of the location the $8 S$ level. This level is the prime candidate to use for an atomic PNC experiment in Fr. Again guided by a quantum defect calculation shown in figure 13, we used a similar technique to that used for the $9 S$ level: Two photon excitation from the $7 S_{1 / 2} \rightarrow 7 P_{3 / 2} \rightarrow 8 S_{1 / 2}$.

We used two different levels, $7 P_{3 / 2}$ or $7 P_{1 / 2}$, for the first step to the $8 S_{1 / 2}$ level (see Fig. 7). Only the $8 S_{1 / 2}, F=13 / 2$ can be reached with an electric dipole allowed transition when the $7 P_{3 / 2}, F=15 / 2$ is the intermediate state. A diode laser operating at $1.7 \mu \mathrm{m}$ with a linewidth of $110 \mathrm{MHz}$ 


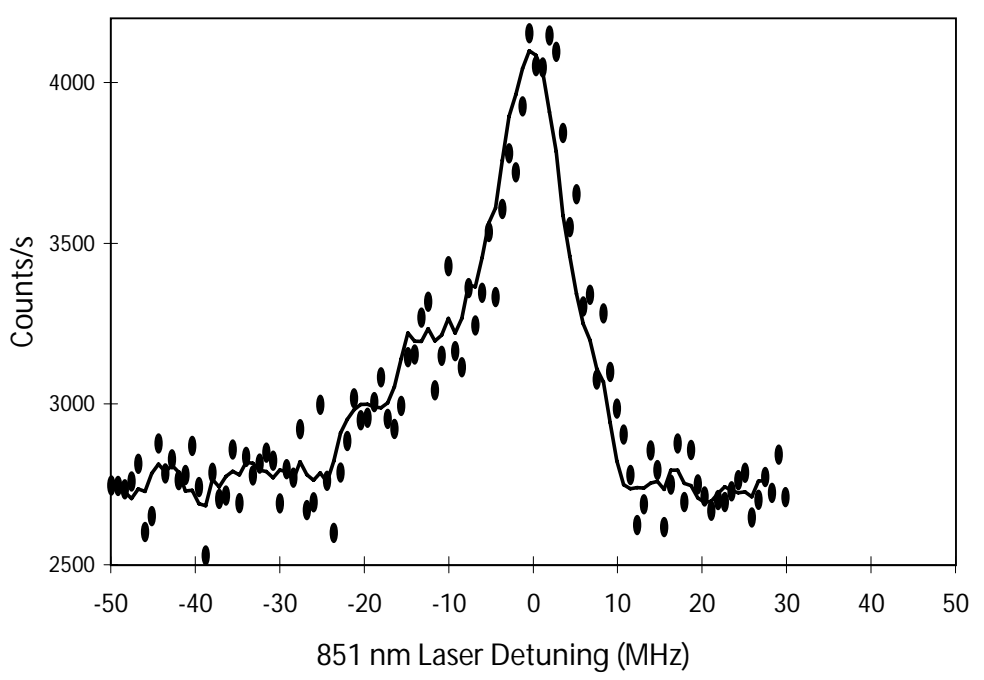

Figure 12. Blue photons from the $9 S$ resonance of Fr as a function of the $851 \mathrm{~nm}$ laser. The zero of the scan corresponds to $11746.978 \pm 0.003 \mathrm{~cm}^{-1}$ The continuous line is a running average to guide the eye.

full width at half maximum (FWHM) excites this transition. Both of the $8 S_{1 / 2}$ hyperfine states can be reached when the $7 P_{1 / 2}, F=13 / 2$ is the intermediate state. A diode laser operating at $1.3 \mu \mathrm{m}$ with a linewidth of $30 \mathrm{MHz}$ FWHM excites this transition. The excitation with the $1.7 \mu \mathrm{m}$ laser allowed us to detect the resonance in two different ways. First we looked for photons at $817 \mathrm{~nm}$ indicating the atom decayed from the $8 S$ level via the $7 P_{1 / 2}$ states. Also when the $1.7 \mu$ m laser resonant to the $7 P_{3 / 2} \rightarrow 8 S_{1 / 2}$ pumps a fraction of the atoms out of the cycling transition decreasing the trap fluorescence accordingly.

The center of gravity energy difference between the $7 S_{1 / 2}$ ground level and the $8 S_{1 / 2}$ level is $19732.523 \pm 0.004 \mathrm{~cm}^{-1}$. The hyperfine separation of the $8 S_{1 / 2}, F=11 / 2$ and $F=13 / 2$ states is $10256 \pm 7 \mathrm{MHz}$ giving a magnetic dipole hyperfine constant $A$ of $1577.8 \pm 1.1 \mathrm{MHz}$ [19].

\subsection{LIFETIME OF THE 7P ELECTRONIC LEVELS}

One of the most stringent tests for the atomic theory is the calculation of the lifetime of an electronic state. For the case of francium and the other alkalis the lifetime of the $\mathrm{D}_{2}$ line linking the $\mathrm{nP}_{3 / 2}$ excited level with the $\mathrm{nS}_{1 / 2}$ ground state is directly proportional to the square of the dipole matrix element between the two levels. The calculation relies in having very good wavefunctions for both the $\mathrm{S}$ and the $\mathrm{P}$ levels. 

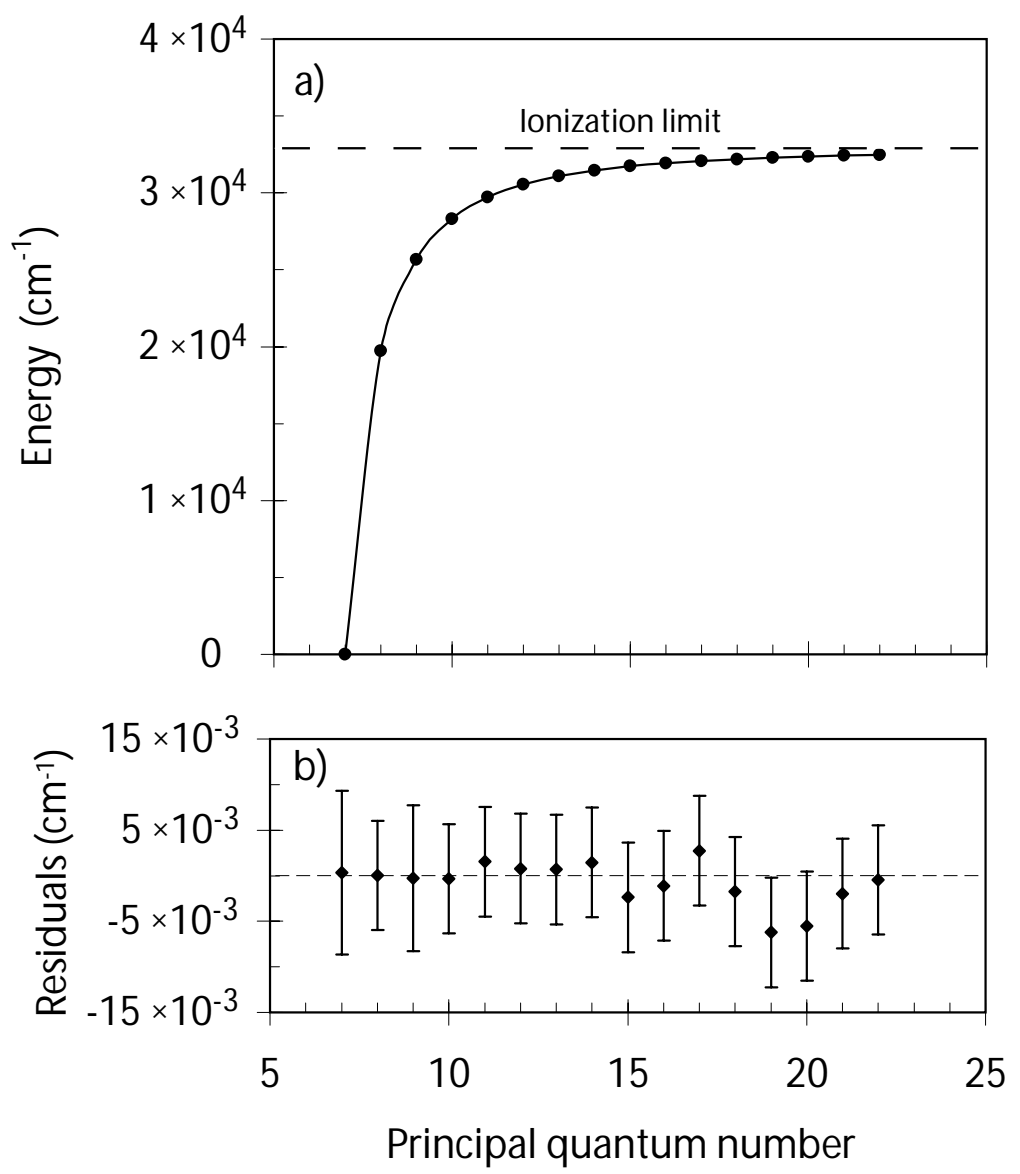

Figure 13. Quantum defect fit to the $S$ series of francium using data from ISOLDE and the $8 S$ and $9 S$ measurements of Stony Brook. b) Residuals of a four parameter quantum defect fit.

There has been considerable interest in measuring radiative lifetimes of the alkali lowest atomic levels to test the $a b$ initio calculations. A result of the activity is the resolution of a prior discrepancy between the theoretical and experimental lifetimes in $\mathrm{Li}[50]$ and $\mathrm{Na}[51,52,54]$. Recent measurements are now in agreement with the calculations. The case of cesium is particularly important. The $\mathrm{D}_{2}$ line lifetime has been under intensive work both experimentally $[55,56]$ and theoretically $[11,10]$. The results bring credibility to the atomic calculations and strengthen the knowledge of the atom. The experiments and theory agree at a little better than $0.5 \%$ accuracy. Dzuba et al. [22] and Johnson et al. [23] have calculated the dipole matrix elements for Fr. The matrix element calculations have an accuracy of $\pm 1.0 \%$ to $\pm 2.0 \%$. The same methods used for Fr have been applied to 
Cs. Our comparison of the measured lifetimes to the calculations test their accuracy in a more complex system.

\subsubsection{Atomic Structure Theory}

The calculations of the groups of New South Wales and Notre Dame [22, 23], agree with our measurements testing their ability to generate appropriate Fr wavefunctions for two of the most important energy levels for an atomic PNC measurement. The effect of the weak force modifies the energy structure by mixing some of the $7 P_{1 / 2}$ and $7 P_{3 / 2}$ levels to the ground state, making the electric dipole transition between the ground state and the first excited $S$ state possible. They use many-body perturbation theory (MBPT) to calculate the atomic structure of francium to a high accuracy. To achieve precise results in the heavy alkali atoms, the interaction between the valence and the core electrons must be calculated. This is the most complicated part of the method.

Dzuba et al. use MBPT to calculate E1 transition amplitudes in Fr. They begin with a relativistic Hartree-Fock (RHF) method to obtain a complete set of electron wave functions. They calculate the single-particle electron wave functions in the effective RHF potential as a perturbation expansion in terms of the difference between the exact and RHF Hamiltonians. The relativistic equations automatically take into account the spinorbit interaction. The time-dependent Hartree-Fock (TDHF) method gives the polarization of the core by the optical field. This changes the radial integral by $-7 \%$.

They calculate the states for the electron outside the core with the single-particle equation using the Brueckner approximation

$$
\left(H_{o}+\Sigma\right) \psi=E \psi
$$

where $H_{o}$ is the relativistic Hartree-Fock-Dirac Hamiltonian, and $\Sigma$ is the self-energy operator. The self-energy operator accounts for the correlation interaction of the valence electron with the core. Correlations arise from the Coulomb interaction between electrons in the many-body system. Dzuba et al. [22] calculate lowest order contributions to $\Sigma$ for Fr. They also consider two classes of higher-order correlations: the hole-particle interaction and the screening of the Coulomb interaction. Screening factors from Cs account for the higher-order correlations in a semiempirical way.

Once they determine $\Sigma$ they find the states of the external electron by iteratively solving Eq. 1 starting with the RHF wave functions. This method accounts for the dominating Brueckner-type correlation diagrams that correct the radial integrals by $\approx-10 \%$. The non-Brueckner correlations are mostly from structural radiation and renormalization of the wave function, that modify the radial integral by $-0.4 \%$. These are extrapolated from sim- 
ilar corrections in Cs. The accuracy of the calculated radial integrals in Fr by Dzuba et al. is expected to be $\pm 1 \%$.

The RHF calculation of Johnson et al. [23] is in good agreement with that of Dzuba et al. The matrix elements of the two groups differ slightly mostly from a difference in the Brueckner-type corrections. Johnson et al. calculated the corrections to third order and included fourth- and higherorder terms empirically. Since these terms play a significant role, it is expected that the result will not be as accurate as their "all-order" calculation in Cs [11]. The accuracy of the calculated matrix elements in Fr by Johnson et al. is expected to be $\pm 2 \%$.

The MBPT calculations predict the location of energy levels, hyperfine structure, E1 transition amplitudes, and parity non-conserving transition amplitudes. These quantities are calculated from the MBPT atomic wave functions. Comparisons with experiments test the accuracy of the wave functions over different ranges of $\mathbf{r}$.

\subsubsection{Measurements of atomic lifetimes in $\mathrm{Fr}$}

The most intuitive way to measure an atomic lifetime is to excite the atoms and record the exponential decay of the fluorescence as a function of time. It is important to have quantitative understanding of the measurement equipment. To measure a time interval, the time scale must be precisely calibrated.

In time-correlated single-photon counting a laser repetitively excites the atoms and a fixed position detector collects the fluorescence as a function of time. The atoms move slowly so that the solid-angle for the fluorescence collection is constant. The method relies on fast electronics to precisely record the time interval for detection events. Count rates are low to prevent dead-time effects in the electronics and to prevent preferential counting of early events. Low particle densities are necessary to minimize radiation trapping and quenching effects due to collisions. Advantages of the method include good statistics and less sensitivity to the divergence of the atomic source.

Our technique to measure lifetimes efficiently utilizes the limited number of available radioactive atoms. Trapped atoms in a MOT are cold and well localized so that each atom can scatter photons over many cycles. Fast electro-optic switches turn the trapping or repumping laser beams on and off to measure the lifetime of the atoms. A fixed photomultiplier tube detects the exponential decay of the fluorescence. Time-correlated singlephoton counting of the fluorescence photons determines the lifetime. The method requires no lasers other than those necessary to trap Fr.

The trapping laser at $718 \mathrm{~nm}$ provides excitation for the $7 p^{2} P_{3 / 2}$ lifetime and passes through two electro-optic modulators (EOM), before entering 
the trap. We use two modulators to turn the light off cleanly and to get a large extinction ratio. The light turns on and off with a Gaussian 1/e (power) fall time of $9 \mathrm{~ns}$ and extinction ratio better than 800:1 (after 30 ns).

The $718 \mathrm{~nm}$ trapping laser is continuously on for the $7 p^{2} P_{1 / 2}$ measurement. A second laser at $817 \mathrm{~nm}$ repumps the atoms on the $7 s^{2} S_{1 / 2}$, $\mathrm{F}=11 / 2 \rightarrow 7 p^{2} P_{1 / 2}, \mathrm{~F}=13 / 2$ transition with total intensity of $17 \mathrm{~mW} / \mathrm{cm}^{2}$. The repumping laser, chopped with the EOMs, provides the excitation for the $7 p^{2} P_{1 / 2}$ lifetime measurement. The extinction ratio of the repumper is better than 250:1 with $20 \mathrm{~mW}$ of laser power. The main trapping laser contributes little background counts due to the $817 \mathrm{~nm}$ interference filter (bandwidth $=10 \mathrm{~nm}$ ) in the detector assembly. See figure 14 for a diagram of the timing sequences for the two measurements.

During normal MOT operation the population in the lower $\mathrm{F}=11 / 2$ ground state is $\approx 10^{3}$ times lower than the $\mathrm{F}=13 / 2$ state. Without transferring population to the lower ground state the fluorescence from the $7 p^{2} P_{1 / 2}$ level is too small to be detected. As a way to actively transfer the population from the $\mathrm{F}=13 / 2$ state to the $\mathrm{F}=11 / 2$ ground state, we use an additional depumper beam tuned to the $7 s^{2} S_{1 / 2}, \mathrm{~F}=13 / 2 \rightarrow 7 p^{2} P_{3 / 2}, \mathrm{~F}=13 / 2$ transition. We derive the depumper from part of the trapping laser beam shifted down in frequency by $600 \mathrm{MHz}$ with a double-passed acousto-optic modulator. The depumper beam has intensity of $0.4 \mathrm{~mW} / \mathrm{cm}^{2}$.

To measure the $7 p^{2} P_{1 / 2}$ lifetime the repumper is on for $4.5 \mu s$ and is off for $4 \mu \mathrm{s}$. While the repumper is off, the depumping laser beam is on. Once significant population moves from the upper to the lower ground state, a short (40 ns) pulse from the repumping laser excites the atoms and we detect fluorescence at $817 \mathrm{~nm}$ for $500 \mathrm{~ns}$ (see Fig.14 b).

A data set consists of accumulated counts with atoms in the trap until the statistics $\left(\approx 1 \times 10^{5}\right.$ fluorescence counts/channel $)$ are adequate which takes about 20 minutes (see Fig. 15 a). We then shift the trapping laser frequency off resonance so that there are no atoms in the trap and collect background for the same length of time. The background spectrum gives precise knowledge of the excitation function for the atoms (see Fig. $15 \mathrm{~b}$ ). The $100 \mathrm{MeV}{ }^{18} \mathrm{O}$ beam is on the target while accumulating the signal and background spectra in case there is background from the radiation associated with the nuclear reaction. The subtraction of the background spectrum from the signal gives the data which is the exponential decay of the fluorescence for $\approx 5$ lifetimes (see Fig. $15 \mathrm{c}$ ).

The statistical quality of the data is evident from Fig. 15. The signalto-noise ratio $20 \mathrm{~ns}$ after the edge of the excitation function is 1200 . For the $7 p^{2} P_{3 / 2}$ lifetime measurement the background count rate is $700 \mathrm{~Hz}$ mostly from dark counts and the imperfect extinction of the $718 \mathrm{~nm}$ laser. 


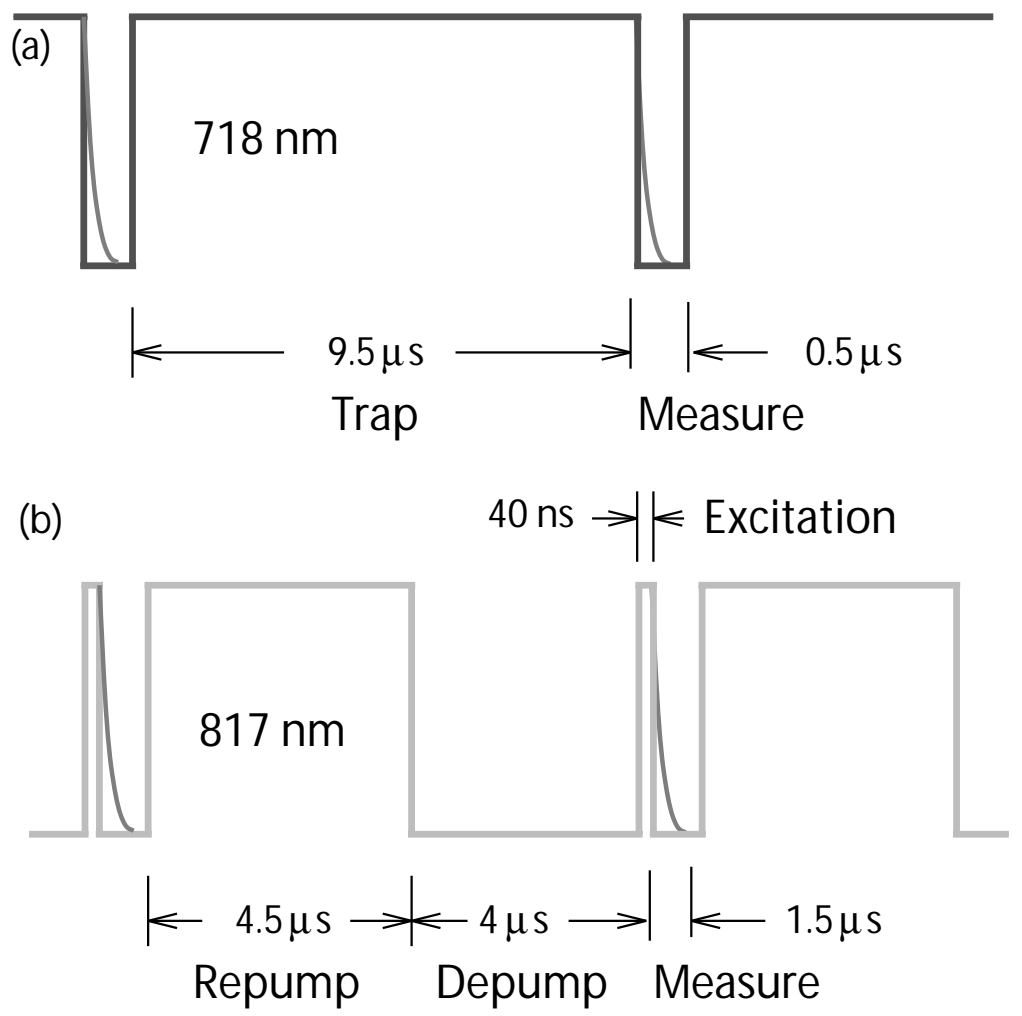

Figure 14. Timing for the lifetime measurements. a) for $D_{2}$ line. b) for $D_{1}$ line.

For the $7 p^{2} P_{1 / 2}$ lifetime measurement the background count rate is $400 \mathrm{~Hz}$ mostly from dark counts and leakage of the $718 \mathrm{~nm}$ light through the 817 nm interference filter.

The data analysis proceeds roughly as follows: We first apply an analytical correction to all data points (sometimes called pulse pileup) arising from the fact that the early events are preferentially counted [58]. Tables 1,2 show the error budget for the two $D$ lines in $\mathrm{Fr}$ and in Rb.

We perform a time calibration of the time to analog converter (TAC) and the multi channel analizer (MCA) and get an uncertainty of $\pm 0.04 \%$. Their amplitude and time linearity, and the frequency jitter of the cycle contribute at most $\pm 0.1 \%$.

To obtain a data sample (trace a in Fig. 15), we take the signal (trace $\mathrm{b}$ in Fig. 15) and subtract the background (trace c in Fig. 15). We then fit the data for a given number of channels, starting at a time where the light is off, to a decaying exponential with a constant background. At the bottom of Fig. 15 are the residuals of such a fit divided by the statistical 

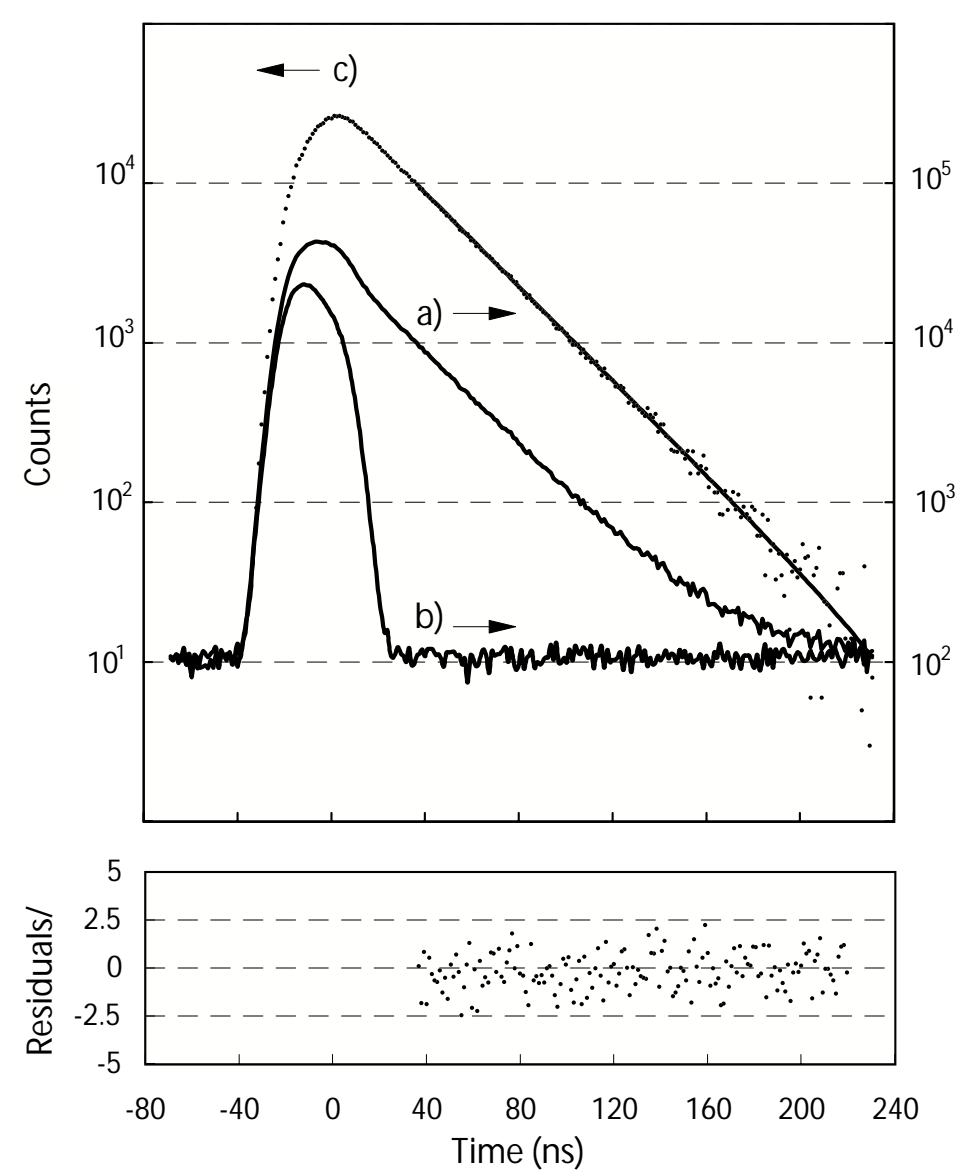

Figure 15. Decay curves of Fr $7 p^{2} P_{1 / 2}$ state. Trace a is the raw data with Fr in the trap and trace $\mathrm{b}$ is the background. Trace $\mathrm{c}$ is the subtraction of a minus $\mathrm{b}$ and the straight line is a pure exponential fit to trace c. The bottom shows the residuals of the fit divided by the statistical uncertainty of each point. The reduced $\chi^{2}=0.98$.

uncertainty of each point.

We also fit the data to the convolution of the well characterized instrument function with a single exponential. The convolution allows us to use points closer to the turn-off time and gives the same value for the lifetime within the statistical uncertainty as that obtained with a single exponential. The value of the constant background from the fits is very small (less than 5 counts), consistent with zero. Although the quality of each individual fit is very good, (reduced $\chi^{2} \approx 1$ ), the particular value obtained for the lifetime depends on the number of channels fitted. For each individual Fr run we change the number of fitted channels both at the begining and at the end of the data sample. We vary the starting point of the fit with 
respect to the light turn-off time by $0.8 \tau$ and the end point by as many as $5 \tau$. We assign an uncertainty that accounts for the variation in the lifetime from the truncation in the fit [56].

The number of atoms in the trap is small $(\mathrm{N} \approx 1000)$ and the typical diameter of the trap is $\leq 1 \mathrm{~mm}$. This minimizes the possibility of multiple absorption. We perform the measurement with different values of the trapping magnetic field gradient and see no change in the measured lifetime.

We have considered the possibility of hyperfine and Zeeman quantum beats, but we have not observed any in the fluorescence signal. The hyperfine splitting between the $F=15 / 2$ and $F=13 / 2$ is $617 \mathrm{MHz}$. The laser linewidth $(\leq 500 \mathrm{kHz})$ is too narrow and the pulse length $(10 \mu \mathrm{s})$ and fall time (9 ns) too long, to excite coherently both states. Zeeman quantum beats are a possible source of systematic shifts. The position dependent force in a MOT requires a gradient of the magnetic field in three dimensions. For our trap an atom that fluoresces may be in a magnetic field of at most 0.5 Gauss. The inhomogenous magnetic field gives each atom a different Larmor frequency, and the different polarizations available from all the laser beams will tend to average out this effect when we count photons without a polarizer in front of the detector. Nevertheless, we have calculated the contribution of a Zeeman quantum beat to the lifetime of ${ }^{210} \mathrm{Fr}$ for a geometry that allows coherences between excited state $m_{F}$ sublevels differing by two (beat frequency $140 \mathrm{kHz}$ ). In our calculation the fitted lifetime is $0.01 \%$ shorter than that from the decay free from quantum beats. We take this number as a systematic error. In order to confirm the absence of other oscillations or correlations in the data we Fourier transform the residuals of the fit and look for any possible remanent frequency. The spectrum shows only noise with no distinguishable peaks.

To search for other possible shifts in the lifetime, we have measured the lifetime of the $5 p^{2} P_{3 / 2}$ level of ${ }^{87} \mathrm{Rb}$ using exactly the same apparatus and technique. With $\mathrm{Rb}$ in the trap, we have varied the number of atoms by decreasing the repumper power. We changed the modulation amplitude of the trapping laser. We moved the position ( \pm one trap diameter) of the trap by imbalancing the light in one arm of the trap, while keeping the detector fixed, and we changed the magnetic field gradient. For all these tests we found no appreciable effect within the statistical error of the lifetime from a single data run. We take this number as a limit on other systematic errors.

Figure 16 shows the results of our measurements in Fr and a comparison with the calculated lifetimes. From our measurements of the atomic lifetimes in Fr we determine the radial matrix elements for the transitions. The excited $7 p^{2} P_{3 / 2}$ and $7 p^{2} P_{1 / 2}$ levels have a single decay mode and the lifetimes are related to a single reduced radial matrix element between the excited and ground levels by: 
TABLE 1. Error budget for the lifetime of the $\mathrm{D}_{2}$ lines of $\mathrm{Rb}$ and $\mathrm{Fr}$ in percentage.

\begin{tabular}{lcc} 
Error & $\mathrm{Rb}(\%)$ & $\mathrm{Fr}(\%)$ \\
\hline Systematic & & \\
TAC-MCA nonlinearity & \pm 0.10 & \pm 0.10 \\
Time calibration & \pm 0.04 & \pm 0.04 \\
Truncation error & \pm 0.24 & \pm 0.60 \\
Zeeman quantum beat & \pm 0.13 & \pm 0.01 \\
$\quad$ Other & \pm 0.23 & \pm 0.38 \\
Total systematic & \pm 0.37 & \pm 0.72 \\
\hline Statistical & \pm 0.07 & \pm 0.24 \\
\hline Sum in quadrature & \pm 0.38 & \pm 0.76
\end{tabular}

TABLE 2. Error budget for the lifetime of the $D_{1}$ lines of $\mathrm{Rb}$ and $\mathrm{Fr}$ in percentage.

\begin{tabular}{|c|c|c|}
\hline Error & $\mathrm{Rb} \mathrm{P}_{1 / 2}(\%)$ & $\operatorname{Fr} \mathrm{P}_{1 / 2}(\%)$ \\
\hline \multicolumn{3}{|l|}{ Systematic } \\
\hline TAC-MCA nonlinearity & \pm 0.05 & \pm 0.05 \\
\hline Time calibration & \pm 0.04 & \pm 0.04 \\
\hline Truncation error & \pm 0.11 & \pm 0.19 \\
\hline Zeeman quantum beat & \pm 0.03 & \pm 0.00 \\
\hline Other & \pm 0.25 & \pm 0.25 \\
\hline Total systematic & \pm 0.28 & \pm 0.32 \\
\hline Statistical & \pm 0.10 & \pm 0.23 \\
\hline \multirow[t]{3}{*}{ Sum in quadrature } & \pm 0.30 & \pm 0.39 \\
\hline & $1=\frac{4}{\omega^{3}}$ & $\left|\left\langle J\|r\| J^{\prime}\right\rangle\right|^{2}$ \\
\hline & $\rightarrow J=\overline{3} \overline{c^{2}}$ & $2 J^{\prime}+1$ \\
\hline
\end{tabular}

where $\omega$ in the transition energy divided by $\hbar, c$ is the speed of light, $\alpha$ is the fine-structure constant, $J^{\prime}$ and $J$ are respectively, the excited and ground state angular momenta, $\tau$ is the excited state lifetime, and $\left|\left\langle J\|r\| J^{\prime}\right\rangle\right|$ is the reduced matrix element. Using the experimentally measured transition energies, the 21.02(15) ns (29.45(11) ns) lifetime gives $5.898(22) a_{\infty}\left(4.277(8) a_{\infty}\right)$ in atomic units for the $7 s^{2} S_{1 / 2} \rightarrow 7 p{ }^{2} P_{3 / 2}$ $\left(7 s^{2} S_{1 / 2} \rightarrow 7 p^{2} P_{1 / 2}\right)$ reduced matrix element, with $a_{\infty}$ the Bohr radius $[18,57]$.

The definition of the radial matrix element used in this work (see eq. 2) is also used by Johnson et al. To convert the matrix elements of Dzuba et 


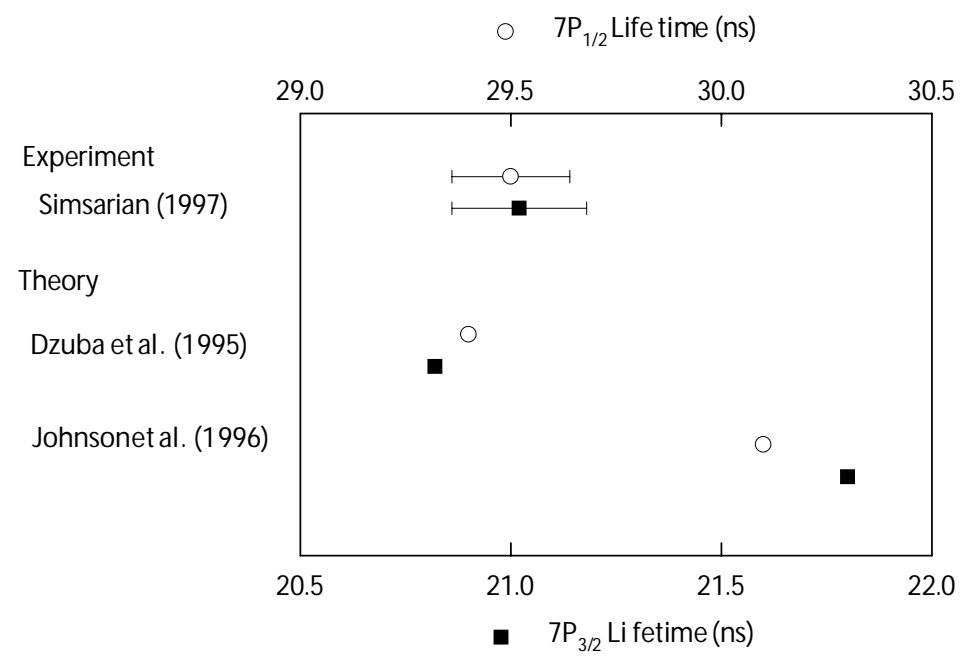

Figure 16. $7 p$ lifetimes of Fr. Experiments and theory.

al. to this convention multiply them by $\sqrt{\left(2 J^{\prime}+1\right) / 3}$.

From the two measurements of lifetime of the $P$ levels we get the atomic line strength ratio. This reveals the large relativistic effects in the heavy Fr atom. The line strength ratio, $\mathbf{S}_{1 / 2} / \mathbf{S}_{3 / 2}$ is the ratio of the reduced matrix elements and is independent of the transition energies. For the nonrelativistic case of the light alkali elements, the ratio is 0.5 . As the relativistic effects become more important in heavier elements the ratio increases. Fig 17 plots the line strength ratio for the alkali. Our result of $0.525(5)$ for $\mathbf{S}_{1 / 2} / \mathbf{S}_{3 / 2}$ in Fr shows a dramatic increase in relativistic effects over Cs. The values of the line strength ratio other than for Fr come from Refs. [52, 53, 56].

According to the simple scaling of the PNC effect as $Z^{3}$, it should be a factor of four larger in Fr than in Cs but the predictions of Dzuba et al. give a factor of eighteen. The extra enhancement comes from relativistic effects. A measure of the relativistic effects is the difference of $\mathbf{S}_{1 / 2} / \mathbf{S}_{3 / 2}$ from 0.5. For Cs the difference is $0.0047(16)$ [55] while in Fr we have measured $0.026(3)[18]$. The ratio of these two numbers accounts for the predicted factor of 18 , indicating the importance of relativistic effects for a PNC experiment.

\subsection{HYPERFINE ANOMALY}

Although there is much information about the distribution of protons within the atomic nucleus, very little is known about the distribution of neutrons in nuclei, and one has to rely heavily on theory. A unique experimental probe of the nuclear magnetization distribution is precision measurements of the 


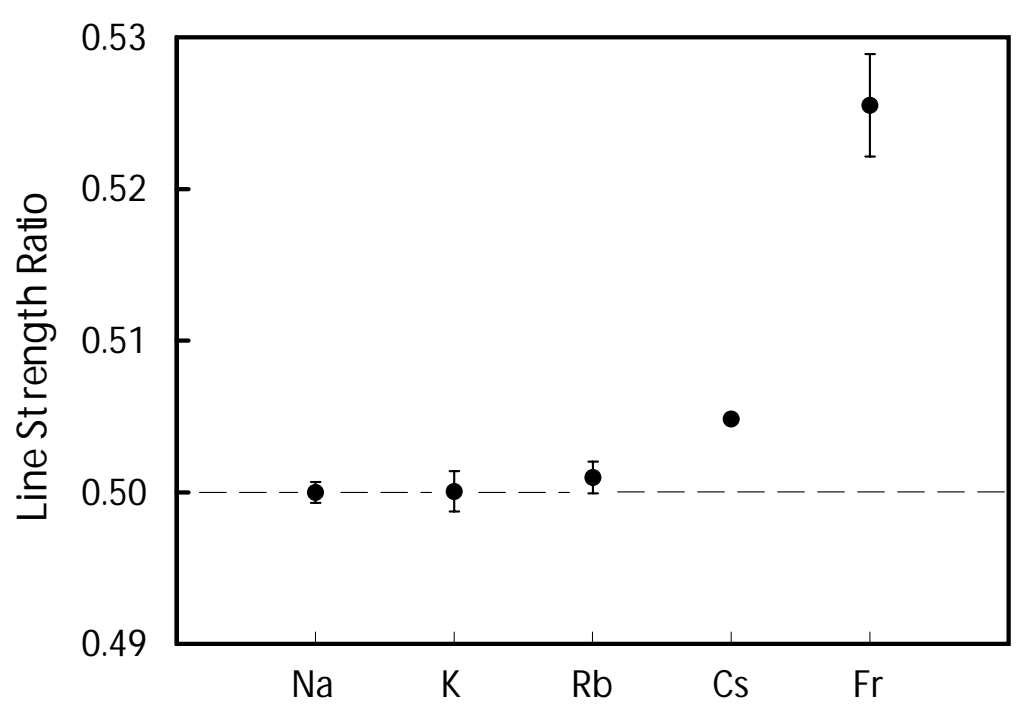

Figure 17. Ratio of line strength of the lowest $P$ levels for alkali atoms. Deviations from 0.5 indicate relativistic effects.

magnetic hyperfine constants $(A)$ with laser spectroscopy. The magnetic hyperfine interaction can be viewed as arising from an effective magnetic field from the electron interacting with the magnetization of the nucleus (see Fig.18). Different atomic states have different radial wavefunctions, and will sample the nuclear magnetization distribution with different weighting. This is the origin of the hyperfine anomaly (Bohr Weisskopf effect). A possible way to get at the neutron positions in nuclei is to look at the radial dependence of the magnetization generated by the neutrons.

Our measurements of the hyperfine structure of the $7 P_{1 / 2}$ level for ${ }^{208-212} \mathrm{Fr}$ have a precision of $300 \mathrm{ppm}$ [59]. These measurements along with previous ground state hyperfine structure measurements reveal a hyperfine anomaly from the Bohr Weisskopf effect [61]. We present in this section some of the experimental aspects that have made this precision possible, and refer the interested reader to the our papers [59,62] on the observation of a hyperfine anomaly and the strong sensitivity of the hyperfine anomaly to the radial distribution of the neutron magnetization.

To obtain a high signal-to-noise ratio out of the few thousand trapped atoms we excite the atoms to the appropriate hyperfine state, and use photon counting techniques. To avoid systematic errors in the calibration of a frequency marker as well as possible slow shifts (tens of minutes) in the laser frequency, we FM modulate the probe laser to generate sidebands separated at about the hyperfine splitting of the $7 P_{1 / 2}$ level $(\approx 6 \mathrm{GHz})$.

The sidebands on the probe laser excite the two hyperfine components 


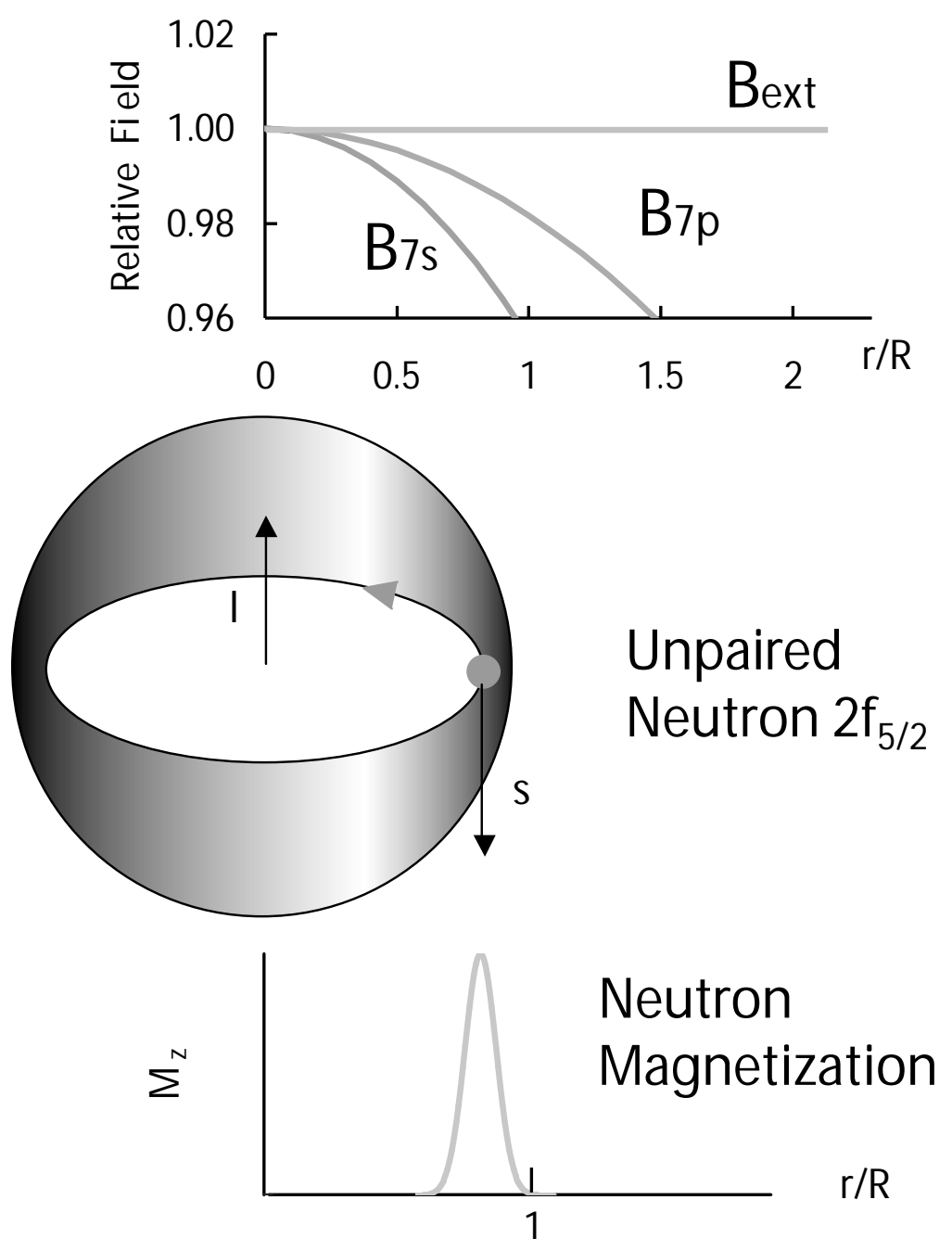

Figure 18. Simplified diagram of the origin of the hyperfine anomaly.

of the $7 P_{1 / 2}$ hyperfine splitting as we scan the carrier. The sidebands span most of the splitting and the carrier only has to scan a small frequency interval to reach the two lines. We repeat the scans with different RF frequencies choosing values to have the sidebands larger or smaller than the hyperfine splitting. Figure 19 shows a series of five scans with changing RF frequency for ${ }^{211} \mathrm{Fr}$. Each scan takes about 5 minutes to complete, with typical steps of $1 \mathrm{MHz} / \mathrm{sec}$.

The signal to noise ratio is larger than 50 in a single scan, so we can determine the peak of each scan with a precision of better than $0.5 \mathrm{MHz}$. 


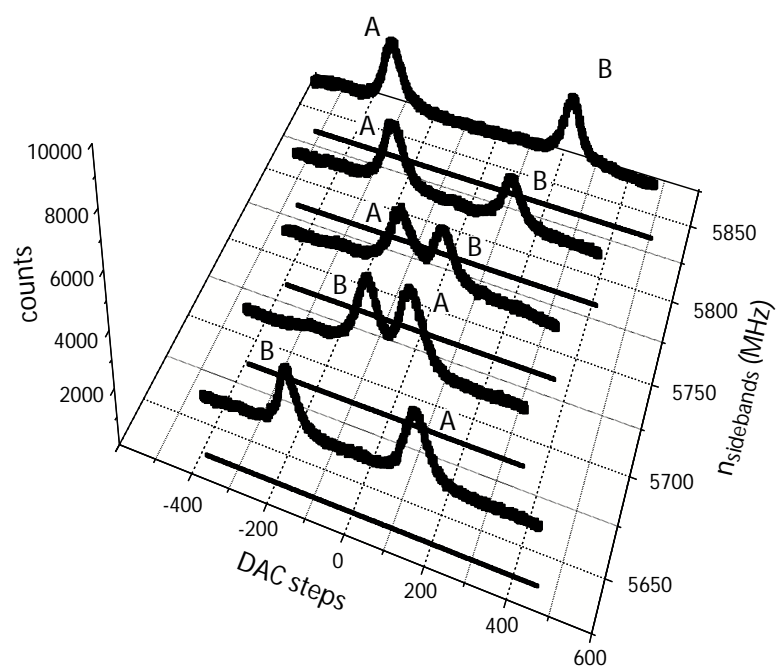

Figure 19. Scans of the $7 P_{1 / 2}, \mathrm{~F}=4$ and $\mathrm{F}=5$ hyperfine states of ${ }^{211} \mathrm{Fr}$. for five different $\mathrm{RF}$ frequencies. A and B identify the appropriate hyperfine state.

We find the splitting by interpolating to zero on a least squares fit of the line positions versus the RF modulation frequency. The RF modulation frequency we obtain is directly the hyperfine splitting.

The method does not rely on an absolute calibration of the scan rate of the laser and how it maps into the DAC steps. It relies on its stability from scan to scan, but the absolute calibration comes from the line intercept as a function of microwave frequency. This frequency is extremely stable and we measure it with a frequency counter.

Figure 20 shows the ratios of the $7 S_{1 / 2}$ to $7 P_{1 / 2}$ hyperfine A constants for a series of isotopes of Fr. There is a distinctive even-odd alternation well beyond the size of our error bars. The qualitative explanation of this observation requires the use of proton and neutron radial distributions [59].

\subsection{THE $7 D$ STATES OF FR}

Our most recent spectroscopic work has been on the $7 D$ states of Fr. See figure 21 for a schematic of the apparatus. These states had not been located before, and we have used very similar techniques as with the $S$ states for both for the estimation of their energy: Quantum defect fits; and for their observation: Double optical resonance spectroscopy. We found the $7 D_{3 / 2}$ and $7 D_{5 / 2}$ levels with a sample of ${ }^{210} \mathrm{Fr}$ atoms confined and cooled in a magneto-optical trap. The upper state of the $7 P_{3 / 2}$ trapping transition serving as the resonant intermediate level to reach the $7 D$ states. We have measured the hyperfine splittings: $\Delta\left(7 D_{3 / 2}, F=15 / 2 \leftrightarrow 13 / 2\right)=$ 


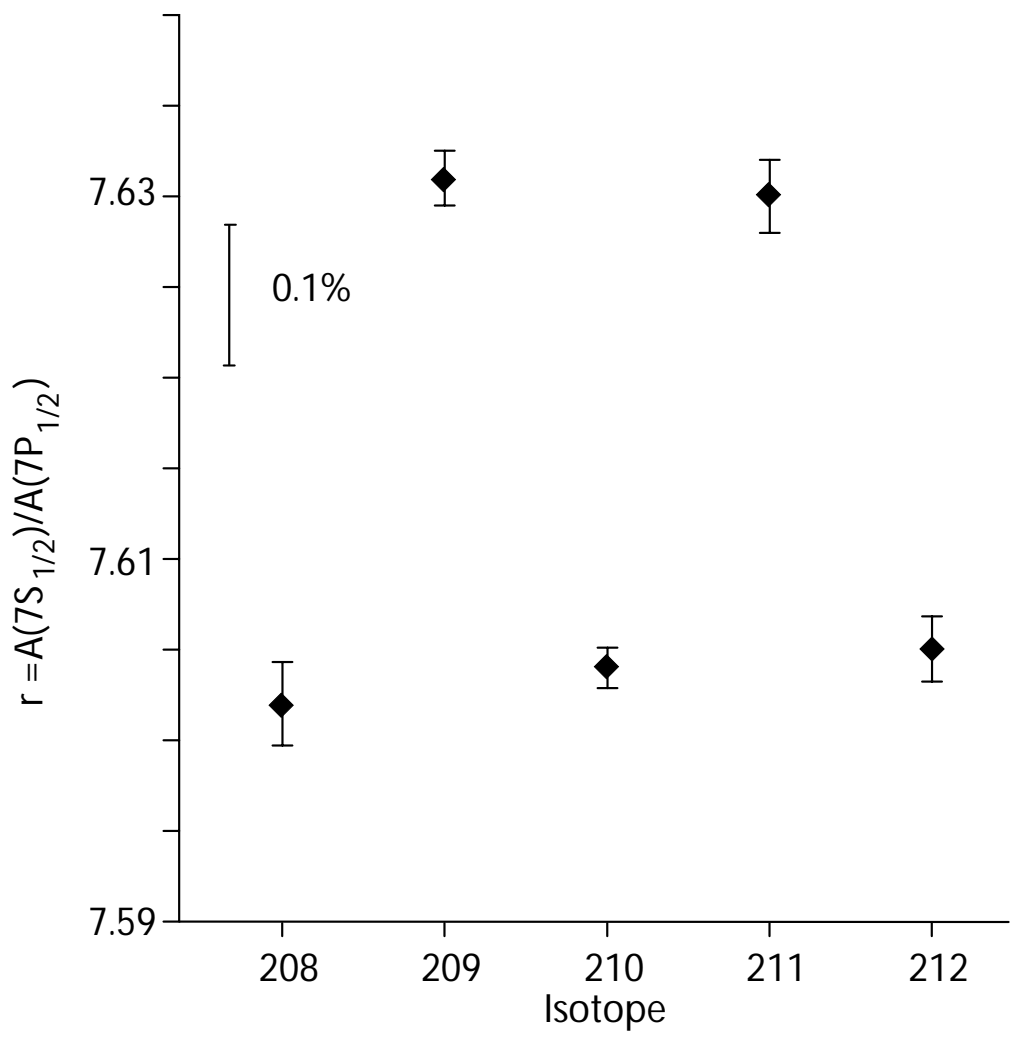

Figure 20. Ratio of hyperfine $A$ magnetic dipole constants of $7 S_{1 / 2}$ and $7 P_{1 / 2}$ states and differential changes observed for five different Fr isotopes. The $S$ states come from the work of Coc et al. [32] and the $P$ states come from our work.

$167 \pm 4 \mathrm{MHz}, \Delta\left(7 D_{5 / 2}, F=17 / 2 \leftrightarrow 15 / 2\right)=-117.5 \pm 2.5 \mathrm{MHz}$, and $\Delta\left(7 D_{5 / 2}, F=15 / 2 \leftrightarrow 13 / 2\right)=-121 \pm 4 \mathrm{MHz}$. Extrapolating the energies of the inaccessible hyperfine levels from the hyperfine constants and assuming $B\left(7 D_{3 / 2}\right)=0$, the center-of-gravity energy difference to the ground state is $E\left(7 D_{3 / 2}\right)=24244.831 \pm 0.003 \mathrm{~cm}^{-1}$ and $E\left(7 D_{5 / 2}\right)=24333.298 \pm$ $0.003 \mathrm{~cm}^{-1}[20]$

We have also measured the lifetime of the $7 D_{3 / 2}$ and $7 D_{5 / 2}$ levels of Fr. We have used a time-correlated single-photon counting technique on a sample of ${ }^{210} \mathrm{Fr}$ atoms confined and cooled in a magneto-optical trap. The upper state of the $7 P_{3 / 2}$ trapping transition serves as the resonant intermediate level for two-photon excitation of the $7 D$ states. A probe laser provides the second step of the excitation, and we detect the decay of the atomic fluorescence. Our measurements help extend the knowledge of this class of atomic wavefunctions in which correlation effects are very significant. We 


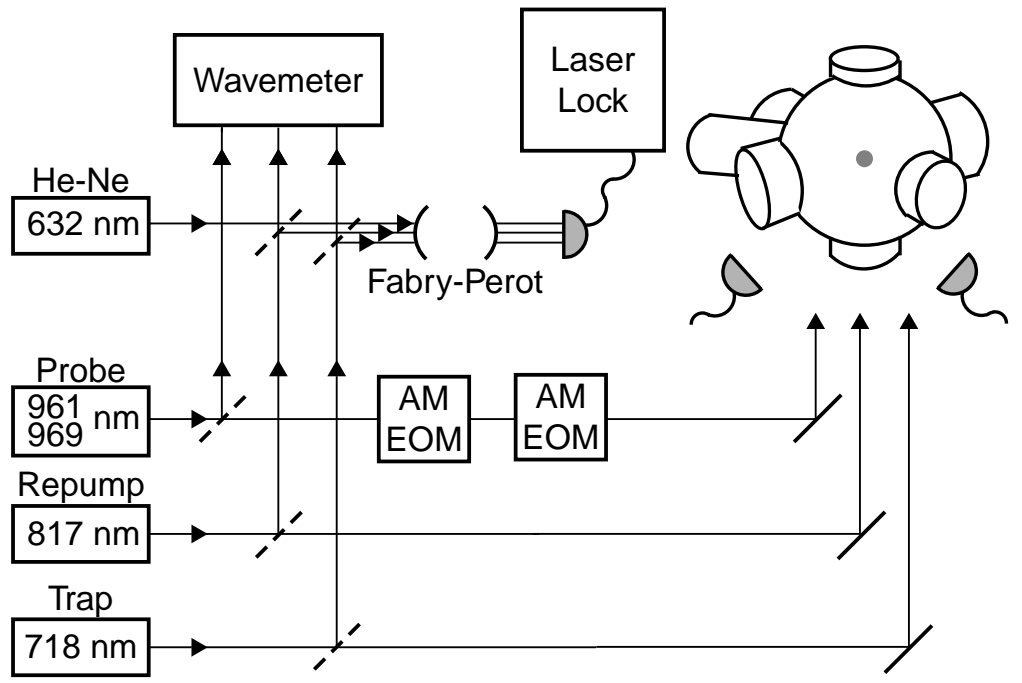

Figure 21. Block diagram of the apparatus for the measurement of the location of the $7 D$ levels.

TABLE 3. Comparison of measured lifetimes with theoretical predictions from semi-empirical calculations and ab initio MBPT calculations of radial matrix elements.

\begin{tabular}{lcc}
\multicolumn{3}{c}{ culations of radial matrix elements. } \\
& $\tau\left(7 D_{3 / 2}\right)[\mathrm{ns}]$ & $\tau\left(7 D_{5 / 2}\right)[\mathrm{ns}]$ \\
\hline This work $\left(\tau\left(7 D_{J}\right)\right)$ & $\mathbf{7 3 . 6} \pm \mathbf{0 . 3}$ & $\mathbf{6 7 . 7} \pm \mathbf{2 . 9}$ \\
Dzuba et al. $[22]$ & 75.4 & 68.7 \\
Safronova and Johnson [63] & 76.0 & 69.5 \\
van Wijngaarden and Xia [64] & 75.9 & 70.3 \\
Biémont et al. $[65]$ & 53 & 77 \\
Theodosiou [66] & 74.5 & 82.7
\end{tabular}

measure lifetimes of $73.6 \pm 0.3 \mathrm{~ns}$ and $67.7 \pm 2.9 \mathrm{~ns}$ for the $7 D_{3 / 2}$ and $7 D_{5 / 2}$ levels, respectively. Table 3 shows a comparison with different theoretical predictions of our lifetime measurements of the $7 d$ levels [21].

\section{Experimental considerations for PNC}

In order to enhance the small parity non-conservation effect in francium it is necessary to perform a measurement based on an 'electroweak interference' between a weak- interaction amplitude $F_{\text {pnc }}$ associated with a $Z^{o}$ exchange, and a parity conserving electromagnetic amplitude $F$ associated with photon exchanges [2]. The means of looking for such an effect consist in preparing a handed experiment, one that can be performed in either a 
right-handed or a left-handed configuration. One measures the transition rate in the two configurations. The results of the two experiments differ by the electroweak interference term. In terms of a right-left asymmetry

$$
A_{\mathrm{RL}}=2 \frac{\operatorname{Re}\left(F F_{\mathrm{pnc}}\right)}{\left|F^{2}+F_{\mathrm{pnc}}^{2}\right|}
$$

The electromagnetic amplitude is much larger than the weak-interaction amplitude and the experiments are designed to make the argument of the numerator real to maximize the effect, so the right-left asymmetry is simply:

$$
A_{\mathrm{RL}}=2 \frac{F_{\mathrm{pnc}}}{F}
$$

Typical numbers for the asymmetry in present cesium experiments are $10^{-6}$. The difficulty of the experiment consists in discriminating the tiny parity violating interference against parity-conserving signals that are many orders of magnitude larger. Systematic errors come from an imperfect reversal of the handedness of the experiment and give false parity violating signals that need to be checked by consistency.

There are two well-established methods to look for the effect. One measures the transition rate for a forbidden transition [3] while the other looks for optical rotation $[4,6]$. Fortson [60] has proposed to look for parity nonconservation in a single ion, but the approach is not applicable to francium. So far, there has been no parity non-conservation measurement performed utilizing the new technologies of laser cooling and trapping. In order to benefit from the cold sample of francium atoms, the transition rate method will be adopted.

In more specific terms we will use a Stark shift to induce a parity conserving amplitude between the $7 \mathrm{~S}$ and $8 \mathrm{~S}$ levels of francium (figure 7 ). The technique follows that used in cesium [3], but modified for francium in a trap. This electromagnetic term will interfere with the weak-interaction amplitude giving rise to a left-right asymmetry as described before.

Francium atoms will accumulate in the MOT. Then, after further cooling to control their velocities, they will be transferred to another region where a non-intrusive trap such as one based on the pure dipole force will keep them ready for the measurement. The steady state number of atoms can be close to $10^{6}$ in the region for the experiment and ultimately depends on the lifetime of the isotope. The measurement is performed by letting the atoms into the mode of a high finesse interferometer tuned to the $7 S$ to $8 S$ transition. This intense laser field can excite the atoms in a region with a coordinate system defined by the external electric field, the external magnetic field and the angular momentum of the photon. If an atom gets excited it will decay via the $7 P$ state. Optical pumping techniques allow one 
to recycle the atom that has performed the parity non-conserving transition many times enhancing the probability to detect the signature photon. Redundancy in the reversal of the coordinates will suppress systematic errors.

It is difficult to predict in quantitative detail all of the systematic problems of this measurement. The most important tool to study and eliminate them is to have an experiment with redundancies.

To estimate the requirements for a parity non-conservation measurement in francium we take the Cs experiment of Prof. Carl Wieman as a guide (See article in [12]). The most important quantity to estimate is the signal to noise ratio since that will determine many of the requirements of the experiment. From the previous discussions on atomic parity nonconservation some kind of interference experiment is needed. The approach works as an amplifier in the full sense of the word, it enlarges the signal, but it also brings noise. The Stark induced part of the signal in photons per second is:

$$
S_{\text {stark }}=\frac{16 \pi^{3}}{3 h \epsilon_{o} \lambda^{3}} E^{2} \beta^{2} I_{o} N
$$

While the parity non-conservation part in photons per second is:

$$
S_{\mathrm{pnc}}=\frac{16 \pi^{3}}{3 h \epsilon_{o} \lambda^{3}} 2 E \beta \operatorname{Im}\left(E_{\mathrm{pnc}}\right) I_{o} N
$$

where $\beta$ is the vector Stark polarizability of the $7 S \rightarrow 8 S$ transition, $E$ is the dc electric field used for the Stark mixing interference, $N$ the number of atoms in the interaction volume, $\lambda$ the wavelength of the transition, $\operatorname{Im}\left(E_{\text {pnc }}\right)$ is the parity non-conservation amplitude expressed as an equivalent electric field, and $I_{o}$ the intensity of the excitation source normalized to the energy of one photon every two lifetimes acting on an area equal to the radiative cross section of the atom. Assuming only shot noise as the dominant source of noise, the signal to noise ratio achieved in one second is:

$$
\frac{S_{\mathrm{pnc}}}{N_{\text {oise }}}=2\left(\frac{16 \pi^{3}}{3 h \epsilon_{o} \lambda^{3}}\right)^{1 / 2} \operatorname{Im}\left(E_{\mathrm{pnc}}\right) \sqrt{I_{o} N}
$$

For francium in the $7 S$ to $8 S$ state, the ratio becomes:

$$
\frac{S_{\mathrm{pnc}}}{N_{\text {oise }}}=7.9 \times 10^{3} \operatorname{Im}\left(E_{\mathrm{pnc}}\right) \sqrt{I_{o} N}
$$

This last expression gives a result in $(\sqrt{\mathrm{Hz}})^{-1}$ when using atomic units for the pnc term. It illustrates where the measurement with francium is stronger: The size of the effect. The calculated value from Dzuba et al. [22] 
for $\operatorname{Im}\left(E_{\text {pnc }}\right)$ of $1.5 \times 10^{-10}$ in atomic units is eighteen times larger than in cesium.

Notice that the ratio does not depend on the particular details of the interference experiment used, that is the value of the vectorial Stark Polarizability of the $7 S \rightarrow 8 S$ transition $\beta$ nor in the particular value of the DC electric field chosen $E$. These factors enter in the signal to noise ratio once the technical noise is considered. The technical noise is any noise whose origin is different from the detection shot noise; it includes intensity noise in the laser, polarization noise, fluctuations in the number of atoms in the interaction region, etc.

Besides starting with a larger effect than in cesium, our efforts are focused on the other two parameters. First the number of atoms N. There should be at least $10^{6}$ atoms in the interacting region.

The second parameter is the normalized intensity of the exciting $7 S$ to $8 S$ laser. Recent technological developments for ultra-low loss mirrors permit the construction of optical cavities with enhancement factors above $10^{5}$. These kind of cavities permit one to store and recirculate many Watts of power.

The efficiency of detection of a $7 S \rightarrow 8 S$ transition can be enhanced by optical pumping techniques. For example, a cycling transition between the $7 S$ and one of the $7 P$ levels could be excited producing many photons. For this reason in the following estimate the efficiency of the detection will be unity, that is, for every atom undergoing a $7 S \rightarrow 8 S$ transition we will assume that at least one photon will be detected. This relaxes the requirements on the light collecting system for the detection of the transition.

Assuming $10^{6}$ atoms and $I_{o}=10^{8}$ (equivalent to $0.27 \mathrm{MW} / \mathrm{cm}^{2}$ ) the signal to noise ratio in one second is:

$$
\frac{S_{\mathrm{pnc}}}{N_{\text {oise }}}=3.7
$$

In francium, isotopes with half-lives larger than 1 minute are separated by up to 16 neutrons. The parity non-conservation effect is 18 times larger than in Cs [22]. In discussions with the atomic theory groups of Flambaum and Johnson they do expect that the atomic theory in Fr can reach the same accuracy as in Cs. Francium can provide a large isotopic difference measurement and a precise single isotope interpretation as well. The potential for a very large signal is balanced by the complication of working with radioactive atoms.

Wieman et al. [67] have explored in great detail all possible combinations not only of electric, magnetic field, and polarization effects that can cause a false PNC signal, but also have taken into account the combinations of the 
gradients of those fields that can give rise to systematic effects. By analyzing their work, the main possible change that we can implement is to change the direction of the DC electric field to make it parallel with the optical cavity axis. Drell and Cummins $[68,69]$ found that in this configuration the $M 1$ transition does not interfere with the PNC term nor with the $\beta E$ Stark induced term, one of the major systematics that they had to study. Drell and Cummings found that for a laser with polarization $\hat{\epsilon}$ oriented at an angle $\theta$ with respect to the $\mathbf{B}$ field, which itself is perpendicular to $\mathbf{E}$ and the $\mathbf{k}$ vector of the excitation laser, the absorption transition probability for each Zeeman component contains a pseudoscalar term proportional to $(\hat{\epsilon} \cdot \mathbf{B})(\hat{\epsilon} \cdot \mathbf{E} \times \mathbf{B})$.

The very high intensities available in a standing wave will exert a repelling force that will tend to move the cold atoms to a region of low intensity. FM modulation at integers of the free spectral range of the cavity can create a slowly moving traveling envelope to solve this problem.

Since all of the available Fr isotopes have hyperfine structure $(\mathbf{I} \neq \mathbf{0})$ measurements of the anapole are possible to extract from the Spin dependent part of the atomic PNC results, just as it has been done with one isotope of Cs $[3,67]$. We expect the anapole moments for the different isotopes to be of the same order than those of Cs or slightly larger it is possible to imagine measuring the anapole moment of all the isotopes to better than $20 \%$ accuracy.

\section{Conlusions}

Francium is a very interesting atom. It has the potential of giving us information about the weak interaction. In particular measurements of the anapole moment in a chain of isotopes will probe weak processes among nucleons. The preparation francium in the laboratory to interrogate it and learn with higher precision its behavior has allowed us to develop new ways to produce it, new ways to capture and trap it, as well as new ways to probe it. We hope that in the future it will give us new information on the weak force.

\section{Acknowledgements}

The work that I have reviewed in this lecture notes is the result of the hard efforts of many people. I would like to particularly acknowledge John. E. Simsarian, Gerald Gwinner, Joshua Grossman, Seth Aubin, Eduardo Gómez, Raymond Fliller III, John A.Behr, Paul Voytas, Wen Zheng Zhao, Matthew R. Pearson, and specially to my Stony Brook colleague Gene D. Sprouse. Thanks to the organizers of the les Houches conference on Trapped particles and fundamental physics Sergei Atutov, Roberto Calabrese and 
Luigi Moi for inviting me to participate. The research has been supported by the United States National Science Foundation and by the United States National Institute of Standards and Technology.

\section{References}

1. Bouchiat M. A. and Bouchiat C., Parity violation in atoms. Rep. Prog. Phys. 60, 1351 (1997).

2. Khriplovich I. B., Parity nonconservation in atomic phenomena,(Gordon and Breach Science Publishers, Philadelphia, 1991.)

3. Wood C. S. , Bennett S. C., Cho D., Masterson B. P. , Roberts J. L. , Tanner C. E. , and Wieman C. E., Measurement of parity nonconservation and an anapole moment in cesium. Science 275, 1759 (1997).

4. Vetter P. A., Meekhof D. M., Majumder P. K., Lamoreaux S. K., and Fortson E. N., Precise test of electroweak theory from a new measurement of parity nonconservation in atomic thallium. Phys. Rev. Lett. 74, 2658 (1995).

5. Budker D., DeMille D., Commins E. D., and Zoltorev M. S., Investigation of nearly degenerate opposite parity states in atomic dysprosium. Phys. Rev. Lett. 70, 3019 (1994).

6. Warrington R. B., Thompson C. D., and Stacey D. N., A new measurement of parity non-conserving optical-roatation at $648 \mathrm{~nm}$ in atomic bismuth. Europhys. Lett. 24, 641 (1993).

7. DeMille D., Parity nonconservation in the $6 s^{2}{ }^{1} S_{0} \rightarrow 6 s 5 d^{3} D_{1}$ transition in atomic ytterbium. Phys. Rev. Lett. 744165 (1995).

8. Fortson E. N., Pang Y., and Wilets L., Nuclear-structure effects in atomic parity nonconservation. Phys. Rev. Lett. 65, 2857 (1990).

9. Pollock S. J., Fortson E. N., and Wilets L., Atomic parity nonconservation: Electroweak parameters and nuclear structure. Phys. Rev. C 46, 2587 (1992).

10. Dzuba V. A. , Flambaum V. V., and Sushkov O. P., Summation of the high orders of perturbation-theory for the parity nonconserving E1-amplitude of the $6 S-7 S$ transition in the cesium atom. Phys. Lett. A 141, 147 (1989).

11. Blundell S. A., Johnson W. R., and Sapirstein J., Relativistic all-order calculations of energies and matrix-elements in cesium. Phys. Rev. A 43, 3407 (1991).

12. Langacker P. editor, Precision tests of the standard electroweak model, (World Scientific, Singapore, 1995).

13. Peskin M. E. , Electroweak reconciliation. Science 281, 1153 (1998).

14. Rosner J. L., Atomic parity violation and precision electroweak physics. An updated analysis. Phys. Rev. D 61, 016006 (1999).

15. Erler J. and Langacker P., Indications for an extra neutral gauge boson in electroweak precision data. Phys. Rev. Lett. 84, 212 (2000).

16. Derevianko A., Reconciliation of the measurement of parity nonconservation in Cs with the standard model. Phys. Rev. Lett. 85, 1618 (2000).

17. Frois B. and Bouchiat M. A. editors, Parity Violations in Atoms and Polarized Electron Scattering, (World Scientific, Singapore, 1999).

18. Simsarian J. E., Orozco L. A., Sprouse G. D., and Zhao W. Z., Lifetime measurement of the $7 p$ levels of francium. Phys. Rev. A 57, 2448 (1998).

19. Simsarian J. E., Zhao W. Z., Orozco L. A., and Sprouse G. D., Two-photon spectroscopy of the francium $8 S_{1 / 2}$ level. Phys. Rev. A 59, 195 (1999).

20. Grossman J. S. , Fliller III R. P., Mehlstäubler T. E., Orozco L. A., Pearson M. R., Sprouse G. D., and Zhao W. Z., Energies and hyperfine splittings of the $7 D$ levels of atomic francium. Phys. Rev. A 62, 052507 (2000).

21. Grossman J. S., Fliller III R. P., Orozco L. A., Pearson M. R., and Sprouse G. D., Lifetime measurements of the 7D levels of atomic francium. Phys. Rev. A 62, 
$062502(2000)$.

22. Dzuba V. A., Flambaum V. V., and Sushkov O. P., Calculation of energy levels, E1 transition amplitudes and parity violation in francium. Phys. Rev. A 51, 3454 (1995).

23. Johnson W. R., Liu Z. W., and Sapirstein J., Transition rates for lithium-like ions, sodium-like ions, and neutral alkali-metal atoms. At. Data Nucl. Data Tables 64, 279 (1996).

24. Safronova M. S., Johnson W. R., and Derevianko A., Relativistic many-body calculations of energy levels, hyperfine constants, electric-dipole matrix elements, and static polarizabilities for alkali-metal atoms. Phys. Rev. A 60, 4476 (1999).

25. Safronova M.S., Johnson W.R., and Derevianko A., High-precision calculation of the parity-nonconserving amplitude in francium. Phys. Rev A 62, 022112 (2000).

26. Sprouse, G. D., Orozco, L .A., Laser trapping of radioactive atoms. Annu. Rev. Nucl. Part. Sci., Vol. 47, 429 (1997).

27. Gwinner G., Laser trapping of radioactive Atoms. (Ph. Dr. Dissertation, SUNY Stony Brook, unpublised, 1995).

28. Simsarian J. E., Laser spectroscopy and lifetime measurements on trapped francium (Ph Dr. Dissertation, SUNY Stony Brook, unpublished, 1998).

29. Perey M., Sur un élément 87, dériveé de l'actinium. C. R. Acad. Sci. 208, 97 (1939).

30. Liberman S., Pinard J., Duong H. T., Juncar P., Vialle J-L., Jacquinot P., Huber G., Touchard F., Büttgenbach S., Pesnelle A., Thibault C., Klapisch R. et Collaboration ISOLDE. First evidence of an optical transition in francium atoms. C. R. Acad. Sci. B 286, 253 (1978).

31. Arnold E., Borchers W., Duong H. T., Juncar P., Lermé J., Lievens P., Neu W., Neugart R., Pellarin M., Pinard J., Vialle J. L., Wendt K., and the ISOLDE Collaboration, Optical laser spectroscopy and hyperfine-structure investigation of the $10^{2} S, 11^{2} S, 8^{2} D$, and $9^{2} D$ excited levels in francium. J. Phys. B 23, 3511 (1990).

32. Coc A., Thibault C., Touchard F., Duong H. T., Juncar P., Liberman S., Pinard J., Lermé J., Vialle J. K., Büttgenbach S., Mueller A. C., Pesnelle A., and the ISOLDE Collaboration, Hyperfine structures and itotpe shifts of Fr 207-213, Fr 220-228; possible evidence of octuplar deformation. Phys. Lett. B 163, 66, (1985).

33. Andreev S. V., Mishin V. I., and Letokhov V. S., Rydberg leves and ionization potential of francium measured by laser resonance ionization in a hot cavity. $J$. Opt. Soc. Am. B 5, 2190 (1988).

34. Simsarian J. E., Ghosh A., Gwinner G., Orozco L. A., Sprouse G. D., and Voytas P. A., Magneto-optic trapping of ${ }^{210}$ Fr Phys. Rev. Lett. 76, 3522 (1996).

35. Lu Z.-T., Corwin K. L., Vogel K. R., Wieman C. E., Dinneen T. P., Maddi J., and Gould H., Efficient collection of Fr into a vapor cell magneto-optical Trap. Phys. Rev. Lett. 79, 994 (1997).

36. Gwinner G., Behr J. A., Cahn S. B., Ghosh A., Orozco L. A., Sprouse G. D., and Xu F., Magneto-optic trapping of radioactive ${ }^{79}$ Rb. Phys. Rev. Lett. 72, 3795 (1994).

37. Lipski A. R., Orozco L. A., Pearson M. R., Simsarian J. E., Sprouse G. D., Zhao W. Z., Gold and isotopically enriched platinum targets for the production of radioactive beams of francium. Nucl. Instr. and Meth. A 438, 217 (1999).

38. Behr J. A., Cahn S. B., Dutta S. B., Ghosh A., Gwinner G., Holbrow C. H., Orozco L. A., Sprouse G. D., Urayama J., and Xu F., A low-energy ion beam from alkali heavy-ion reaction products. Nucl. Instr. and Meth. A 351, 256 (1994).

39. Monroe C., Shann W., Robinson H., and Wieman C., Very cold trapped atoms in a vapor cell. Phys. Rev. lett. 65, 1571, (1990).

40. Lu Z.-T., Bowers C. J., Freedman S. J., Fujikawa B. K., Morata J. L., Shang S.-Q., Coulter K. P., and Young L., Laser trapping of short lived radioactive isotopes. Phys. Rev. Lett. 72, 3791 (1994).

41. Bergeman T., Erez G., and Metcalf H. J., Magnetostatic trapping fields for neutral atoms.Phys. Rev. A35, 1535 (1987).

42. Swenson D. R. and Anderson L. W., Relaxation rates for optically pumped Na vapor 
on silicone surfaces. Nucl. Instr. and Meth. B29, 627 (1988).

43. Stephens M., Rhodes R., and Wieman C., Study of wall coatings for vapor-cell laser traps. J. Appl. Phys. 76, 3479 (1994).

44. Goldenberg H. M., Kleppner D., and. Ramsery N. F., Atomic beam resonance experiments with stored beams. Phys. Rev. 123, 530 (1961).

45. Vanier J., Simard J. F., and Boulanger J. S., Relaxation and frequency-shifts in ground state of ${ }^{85} \mathrm{Rb}$. Phys. Rev A, 91031 (1974).

46. Gesternkorn S., Vergès J., and Chevillard J., Atlas du spectre d'absorption de la moleécule d'iode Part I 10,000-14,000 1/cm (Editions du Centre national de la recherche Scientifique, Paris, 1978).

47. Zhao W. Z., Simsarian J. E., Orozco L. A., and Sprouse G. D., A computer-based digital feedback control of frequency drift of multiple lasers. Rev. Sci. Instrum. 69, 3737 (1998).

48. Bauche J., Duong H. T., Juncar P., Liberman S., Pinard J., Coc A., Thibault C., Touchard F., Lermé J., Vialle J. L., Büttgenbach S., Mueller A. C., Pesnelle A., and the ISOLDE collaboration, Accurate wave-numbers of the 1st resonance doublet of francium atoms and relativistic contribution to isotope shifts in the resonance doublets of francium an cesium atoms. J. Phys. B 19, L593 (1986).

49. Simsarian J. E., Shi W., Orozco L. A., Sprouse G. D., Zhao W. Z., Two-photon spectroscopy of trapped francium $7 S-1 / 2 \rightarrow 9 S_{1 / 2}$. Opt. Lett. 21, 1939 (1996).

50. McAlexander W. I., Abraham E. R. I., and Hulet R. G., Radiative lifetime of the 2P state of lithium. Phys. Rev. A 54, R5 (1996).

51. Oates C. W., Vogel K. R., and Hall J. L., High precision linewidth measurement of laser-cooled atoms: Resolution of the $\mathrm{Na} 3 p^{2} P_{3 / 2}$ lifetime discrepancy. Phys. Rev. Lett. 76, 2866 (1996).

52. Volz U., Majerus M., Liebel H., Schmitt A., and Schmoranzer H., Precision lifetime measurements on NaI $3 p^{2} P_{1 / 2}$ and $3 p^{2} P_{3 / 2}$ by beam-gas-laser spectroscopy. Phys. Rev. Lett. 76, 2862 (1996).

53. Volz U. and Schmoranzer H., Precision lifetime measurements on alkali atoms and on helium by beam-gas-laser spectroscopy. Physica Scripta T65, 48 (1996).

54. Jones K. M., Julienne P. S., Lett P. D., Phillips W. D., Tiesinga E., and Williams C. J., Measurement of the atomic $\mathrm{Na}(3 \mathrm{P})$ lifetime and of retardation in the interaction between two atoms bound in a molecule. Europhys. Lett. 35, 85 (1996).

55. Rafac R. J., Tanner C. E., Livingston A. E., Kukla K. W., Berry H. G., and Kurtz C. A., Precision lifetime measurements of the $6 p^{2} P_{1 / 2,3 / 2}$ states in atomic cesium. Phys. Rev. A 50, R1976 (1994).

56. Young L., Hill III W. T., Sibener S. J., Price S. D., Tanner C. E., Wieman C. E., and Leone S. R., Precision lifetime measurements of Cs $6 p^{2} P_{1 / 2}$ and $6 p^{2} P_{3 / 2}$ levels by single-photon counting. Phys. Rev. A 50, 2174 (1994).

57. Zhao W. Z., Simsarian J. E., Orozco L. A., Shi W. and Sprouse G. D., Measurement of the $7 p^{2} P_{3 / 2}$ level lifetime in atomic francium. Phys. Rev. Lett. 78, 4169 (1997).

58. O'Connor D. V. and Phillips D., Time correlated single photon counting (Academic, London, 1984).

59. Grossman J. S., Orozco L. A., Pearson M. R., Simsarian J. E., Sprouse G. D., and Zhao W. Z., Hyperfine anomaly measurements in francium isotopes and the radial distribution of neutrons. Phys. Rev. Lett. 83, 935 (1999).

60. Fortson E. N., Possibility of measuring parity nonconservation with a single trapped atomic ion. Phys. Rev. Lett. 70, 2383 (1993).

61. Bohr A. and Weisskopf V. F., The influence of nuclear structure on the hyperfine structure of heavy elements. Phys. Rev. 7794 (1950).

62. Grossman J. S., Orozco L. A., Pearson M. R., and Sprouse G. D., Spectroscopy of francium isotopes. Physica Scripta T86, 16 (2000).

63. Safronova M. S. and Johnson W. R. private communication (2000).

64. Van Wijngaarden W. A., and Xia J., Lifetimes and polarizabilities of low lying S, $\mathrm{P}$ and D states of francium. J. Quant. Spectrosc. Radiat. Transfer 61, 557 (1999). 
65. Biémont E., Quinet P., and Van Renterghem V., Theoretical investigation of neutral francium. J. Phys. B 31, 5301 (1998).

66. Theodosiou C. E., Transition probabilities, oscillator strengths, branching ratios and lifetimes of all known states of francium. Bull. Am. Phys. Soc. 39, 1210 (1994); private communication (1993).

67. Wood C. S., Bennett S. C., Roberts J. K., Cho D., and Wieman C. E., Precision measurement of parity nonconservation in cesium. Can. J. Phys. 777 (1999).

68. Drell P. S. and Commins E. D., Parity nonconservation in atomic thallium. Phys. Rev. Lett. 53, 968 (1984).

69. Drell P. S. and Commins E. D., Parity nonconservation in atomic thallium. Phys. Rev. A 32, 2196 (1985). 